\title{
A mechanistic model for airborne and direct human-to-human transmission of COVID-19: effect of mitigation strategies and immigration of infectious persons
}

\author{
Saheb Pal ${ }^{1, a_{(D)}}$ and Indrajit Ghosh ${ }^{2, b_{(D)}}$ \\ 1 Department of Mathematics, Visva-Bharati, Santiniketan 731235, India \\ 2 Department of Computational and Data Sciences, Indian Institute of Science, Bangalore, Karnataka 560012, India
}

Received 19 October 2021 / Accepted 18 December 2021 / Published online 13 January 2022 (C) The Author(s), under exclusive licence to EDP Sciences, Springer-Verlag GmbH Germany, part of Springer Nature 2022

\begin{abstract}
The COVID-19 pandemic is the most significant global crisis since World War II that affected almost all the countries of our planet. To control the COVID-19 pandemic outbreak, it is necessary to understand how the virus is transmitted to a susceptible individual and eventually spread in the community. The primary transmission pathway of COVID-19 is human-to-human transmission through infectious droplets. However, a recent study by Greenhalgh et al. (Lancet 397:1603-1605, 2021) demonstrates 10 scientific reasons behind the airborne transmission of SARS-COV-2. In the present study, we introduce a novel mathematical model of COVID-19 that considers the transmission of free viruses in the air beside the transmission of direct contact with an infected person. The basic reproduction number of the epidemic model is calculated using the next-generation operator method and observed that it depends on both the transmission rate of direct contact and free virus contact. The local and global stability of disease-free equilibrium (DFE) is well established. Analytically it is found that there is a forward bifurcation between the DFE and an endemic equilibrium using central manifold theory. Next, we used the nonlinear least-squares technique to identify the best-fitted parameter values in the model from the observed COVID-19 mortality data of two major districts of India. Using estimated parameters for Bangalore urban and Chennai, different control scenarios for mitigation of the disease are investigated. Results indicate that the vaccination of susceptible individuals and treatment of hospitalized patients are very crucial to curtailing the disease in the two locations. It is also found that when a vaccine crisis is there, the public health authorities should prefer to vaccinate the susceptible people compared to the recovered persons who are now healthy. Along with face mask use, treatment of hospitalized patients, and vaccination of susceptibles, immigration should be allowed in a supervised manner so that economy of the overall society remains healthy.
\end{abstract}

\section{Introduction}

The current outbreak of coronavirus disease 2019 (COVID-19) has significantly affected public health and the economy worldwide. As of 21 November 2021, more than 257 million COVID-19 cases and 5 million deaths have been reported globally [6]. COVID-19 is an emerging respiratory infectious disease that was first detected in early December 2019 in Wuhan, China. The virus that causes COVID-19 is severe acute respiratory syndrome coronavirus 2 (SARS-CoV-2), which spread rapidly throughout the globe. The World Health Organization (WHO) initially declared the COVID-19 outbreak as a public health emergency of international concern but with the spread across and within countries leading to a rapid increase in the number of cases, the

\footnotetext{
${ }^{a}$ e-mail: saheb92.math@gmail.com

b e-mail: indra7math@gmail.com (corresponding author)
}

outbreak was officially declared a global pandemic on 11 March 2020 [47]. Given the global scenario on the series of waves and unprecedented strains of COVID19 , there is a need for more investigation to timely and effectively curtail the spread of the disease.

Mathematical modelling is a very versatile and effective instrument for understanding infectious disease transmission dynamics and helps us to develop preventive measures for controlling the infection spread both qualitatively and quantitatively. To model the epidemiological data of infectious diseases, compartmental models are widely used due to their simplicity and wellstudied applications [18]. In this regard, the recent epidemiological threat of COVID-19 is not an exception. In such types of models, the population is categorized into several classes, or compartments, based on the stage of the infection that is affecting them. These models are governed by a system of ordinary differential equations that take into account the time-discoursed infection sta- 
tus of the population compartments. Wu et al. [49] first developed a mathematical model for COVID-19 which is based on Susceptible-Exposed-Infectious-Recovered (SEIR) model and explains the transmission dynamics and estimate the national and global spread of COVID19. After that, various mathematical models were proposed by considering several stages of susceptible and infected populations [19,36]. Many researchers studied the dynamics of COVID-19 using real incidence data of the affected countries and examined different characteristics of the outbreak as well as evaluated the effect of intervention strategies implemented to suppress the outbreak in respective countries $[14,27,41]$. In addition, a comparative analysis of the most affected countries during the second wave of COVID-19 was done by Easwaramoorthy et al. [10] using fractal-based prognostic model.

Presently, the second wave of COVID-19 is affecting most of the countries in the world. In a densely populated country like India, this scenario is more threatening. On April 15, 2021, the daily cases of COVID-19 were double the first peak. However, The epidemic evolution of the country is quite complex due to regional inhomogeneities. Thus, governments of various states in India are adopting different strategies to control the outbreak, such as wearing masks, vaccination drives, social distancing guidelines, partial lockdowns, and restricted store hours in place. Thus, gaining an understanding of how this outbreak spread in the form of waves and possible interventions on a shortterm basis is urgent. As of 21 November 2021, more than 34 million COVID-19 cases and more than 465 thousand deaths are reported in India [7]. We have chosen to concentrate on two major districts namely, Bangalore urban and Chennai of India for our case studies. These two districts are very crucial hubs of south India and are experiencing a severe second wave of COVID19. Additionally, taking small regions is also necessary to make reliable projections using mechanistic mathematical models. Due to the small area and populations, it is also easy to implement and supervise specific control measures.

As a case study in India, several researchers proposed models that are fitted to the daily COVID-19 cases and deaths, and examined different control strategies $[13,15,17,19,28,34,36]$. Some of the studies explore the vaccine allocation strategy in India due to limited supply and to support relevant policies [12]. Regarding the upcoming wave of COVID-19 in India, Kavitha et al. [17] discussed the epidemic rate in the provinces of India by considering the SIR and fractal models and notifying that there is a possibility of a third wave during the first week of August 2021. However, none of the above studies has considered the airborne transmission of the SARS-COV-2 virus in their respective models with application to Indian districts.

To control a pandemic outbreak, it is necessary to understand how the virus transmits to a susceptible individual and eventually spreads in the community. The primary transmission pathway of COVID19 is human-to-human propagation through infectious droplets [45]. However, a recent study by Greenhalgh et al. [16] suggests that SARS-COV-2 can be transmitted through the air and they showed 10 scientific reasons behind this. Later on, Addleman et al. [1] commented that Canadian public health guidance and policies should be updated to address the airborne mode of transmission. The authors also suggested that addressing airborne transmission requires the expertise of interdisciplinary teams to inform solutions that can end this pandemic faster. Motivated by these scientific pieces of evidence, we consider a compartmental model of SEIRtype including shedding of free virus in the air by infectious persons. To the best of our knowledge, this is the first modelling study that considers the airborne transmission of COVID-19 with applications to Indian districts. We consider that the susceptible population becomes exposed in two different ways: firstly through direct contact with an infected population or touches a surface that has been contaminated. This transmission also occurs through large and small respiratory droplets that contain the virus, which would occur when near an infected person. The next is through the airborne transmission of smaller droplets and particles that are suspended in the air over longer distances and time [11]. We also study the effect of immigration along with popular control strategies. The main focus of our study is to explore the following epidemiological issues:

- Mathematically analyze the COVID-19 transmission dynamics by incorporating the airborne pathway of free SARS-CoV-2 virus into the model structure.

- Impact of anti-COVID drugs on the reduction of hospitalized persons.

- Individual and combined effects of various control measures (use of face mask, vaccination) as well as immigration of infectious persons on the COVID-19 pandemic.

The remainder of this paper is organized as follows. In the next section, we propose a deterministic compartmental model to describe the disease transmission mechanism. We consider the amount of free virus in the air as a dynamic variable. Section 3 describes the theoretical analysis of the model, which incorporates the existence of positive invariance region, boundedness of solutions, computation of the basic reproduction number, and stability of disease-free equilibrium. Also, in this section, the existence of forward bifurcation of the model system is explored. In Sect. 4, we fit the mathematical model using nonlinear least-squares technique from the observed mortality data and estimate unknown parameters. Section 5 describe several control mechanisms and immigration of infectives through numerical simulation. We examine the effects of vaccination, treatment by drugs, and use of face masks with different degrees of efficacy as intervention strategies. Finally, in Sect. 6 we discuss the findings and some concluding remarks about mitigation strategy obtained from our study. 


\section{Model description}

To design the basic mathematical model for COVID19 transmission dynamics, some general epidemiological factors are considered including the airborne transmission of the free virus. Several studies of novel coronavirus suggest that an infected person can be asymptomatic (infectious but not symptomatic) or symptomatic with small, moderate, severe, or critical symptoms $[23,46,50,51]$. For simplicity of model formulation, we break the infected individuals into two separated classes, notified and un-notified. Here, the un-notified class contains untested asymptomatic, asymptomatic who are tested negative, untested symptomatic, and symptomatic who are tested negative, whereas notified class contains those symptomatic and asymptomatic persons who are tested COVID-19 positive. Population among both the notified and un-notified class can be hospitalized, when they have a critical health situation. Thus the entire population can be stratified into six main compartments based on the status of the disease: susceptible $(S(t))$, exposed $(E(t))$, un-notified $\left(I_{u}(t)\right)$, notified $\left(I_{n}(t)\right)$, hospitalized $\left(I_{h}(t)\right)$, and recovered $(R(t))$, at time $t$. Consequently, the total population size is given by $N(t)=$ $S(t)+E(t)+I_{u}(t)+I_{n}(t)+I_{h}(t)+R(t)$. In addition, $V(t)$ describes the amount of free virus in the environment.

Now, we consider individuals from each human class have a per capita natural mortality rate $\mu$. The net influx rate of susceptible population per unit time is $\Pi$. This parameter includes new births, immigration, and emigration of susceptible persons. Also, the susceptible population decrease after infection, acquired through the direct contact of notified or un-notified infected individuals. Let $\beta_{1}$ be the transmission rate for direct contact with the modification factor $\nu$ for notified infected individuals. Then $\nu \in(0,1)$, since the notified persons are advised to take preventive measures like face mask use, social distancing more seriously. In addition, the susceptible population is infected through the contact of the free virus in air $[1,16]$. Let $\beta_{2}$ be the transmission rate for free virus contact. The interaction of susceptible individuals with infected individuals (un-notified or notified) follows standard mixing incidence and with free virus follows mass action incidence. For basic details and difference between standard mixing incidence and mass action incidence, interested readers are referred to the third chapter of Martcheva's book [29]. Then the differential equation that describes the rate of change of susceptible individuals at time $t$, is given by

$$
\frac{\mathrm{d} S}{\mathrm{~d} t}=\Pi-\frac{\beta_{1} S\left(I_{u}+\nu I_{n}\right)}{N}-\beta_{2} S V-\mu S+\theta R .
$$

Here, $\theta$ be the rate at which recovered individuals eventually lose the temporal immunity from the infection and become susceptible $[12,35]$.
Alongside, the exposed population $(E(t))$ who are infected individuals, however still not infectious for the community. By making successful contact with infectives, susceptible individuals become exposed. Also, these people decrease at a rate $\gamma$ and become notified or un-notified. The probability that exposed individuals progress to the notified infectious class is $p$ and that of the un-notified class is $1-p$. This assumption leads to the following rate of change in $E$ :

$$
\frac{\mathrm{d} E}{\mathrm{~d} t}=\frac{\beta_{1} S\left(I_{u}+\nu I_{n}\right)}{N}+\beta_{2} S V-(\gamma+\mu) E .
$$

The un-notified individuals $\left(I_{u}\right)$, who are transferred from exposed class at a rate $(1-p) \gamma$ are the population who are un-notified from COVID-19 disease. Also, the un-notified individuals progress to hospitalized class at a rate $\eta_{u}$ and recovered at a rate $\sigma_{u}$. The un-notified individuals are assumed to have no disease-induced mortality rate as most of them will not develop severe symptoms. However, if some of the un-notified persons develop sudden severe symptoms, they will be transferred to the hospital and die as a hospitalized patients. Thus, the equation governing the rate of change of un-notified persons is

$$
\frac{\mathrm{d} I_{u}}{\mathrm{~d} t}=(1-p) \gamma E-\left(\eta_{u}+\sigma_{u}+\mu\right) I_{u} .
$$

The notified individuals $\left(I_{n}\right)$, who are transferred from exposed class at a rate $p \gamma$ are the population who are notified as COVID-19 positive people. Also, the notified individuals reduced by progressing to hospitalized class at a rate $\eta_{n}$, by recovery at a rate $\sigma_{n}$, and COVID-19 induced mortality rate $\delta_{n}$. Therefore, we have the following equation

$$
\frac{\mathrm{d} I_{n}}{\mathrm{~d} t}=p \gamma E-\left(\eta_{n}+\sigma_{n}+\mu+\delta_{n}\right) I_{n} .
$$

The hospitalized individuals $\left(I_{h}\right)$ increase from unnotified or notified people at rates $\eta_{n}$ or $\eta_{u}$, respectively. Also, this class of people recover at a rate $\sigma_{h}$ and decrease through COVID-19 induced mortality rate $\delta_{h}$. Thus, we have the following rate of change of $I_{h}$ :

$$
\frac{\mathrm{d} I_{h}}{\mathrm{~d} t}=\eta_{u} I_{u}+\eta_{n} I_{n}-\left(\sigma_{h}+\mu+\delta_{h}\right) I_{h} .
$$

The recovered individuals $(R)$ increase by recovering from each of the infected classes (un-notified, notified and hospitalized) at rates $\sigma_{u}, \sigma_{n}$, and $\sigma_{h}$ respectively. As mentioned earlier, $\theta$ is the rate at which the recovered population loses immunity against COVID-19 and 


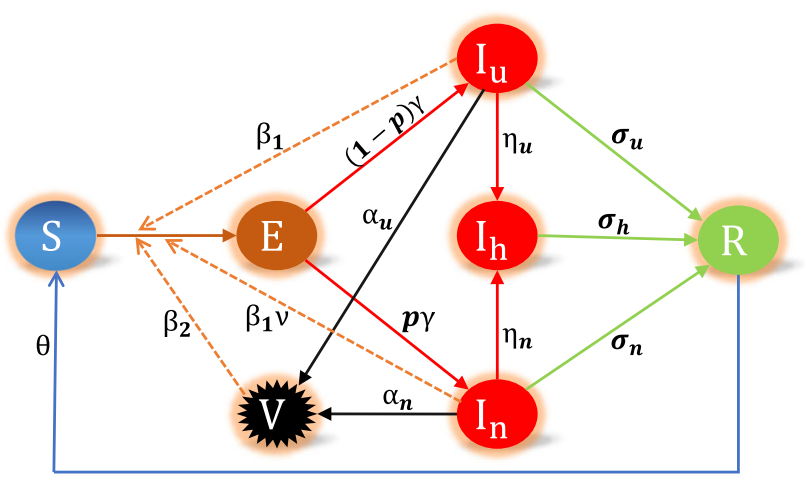

Fig. 1 Schematic diagram of the proposed model. Solid arrows represent the transmission rate from one compartment to other, whereas dashed arrows represent the interaction between compartments

becomes susceptible again. Thus, the following differential equation leads to the rate of change of $R$ :

$$
\frac{\mathrm{d} R}{\mathrm{~d} t}=\sigma_{u} I_{u}+\sigma_{n} I_{n}+\sigma_{h} I_{h}-(\theta+\mu) R
$$

The differential equation describing the rate of change of free virus, increased from the shedding rate of unnotified and notified patients at rates $\alpha_{u}$ and $\alpha_{n}$, respectively with a natural clearance rate $\mu_{c}$. Thus

$$
\frac{\mathrm{d} V}{\mathrm{~d} t}=\alpha_{u} I_{u}+\alpha_{n} I_{n}-\mu_{c} V .
$$

Figure 1 describes the flow patterns of individuals between compartments over time.

Assembling all the above differential equations, we have the following system

$$
\left\{\begin{array}{l}
\frac{\mathrm{d} S}{\mathrm{~d} t}=\Pi-\frac{\beta_{1} S\left(I_{u}+\nu I_{n}\right)}{N}-\beta_{2} S V-\mu S+\theta R \\
\frac{\mathrm{d} E}{\mathrm{~d} t}=\frac{\beta_{1} S\left(I_{u}+\nu I_{n}\right)}{N}+\beta_{2} S V-(\gamma+\mu) E \\
\frac{\mathrm{d} I_{u}}{\mathrm{~d} t}=(1-p) \gamma E-\left(\eta_{u}+\sigma_{u}+\mu\right) I_{u} \\
\frac{\mathrm{d} I_{n}}{\mathrm{~d} t}=p \gamma E-\left(\eta_{n}+\sigma_{n}+\mu+\delta_{n}\right) I_{n} \\
\frac{\mathrm{d} I_{h}}{\mathrm{~d} t}=\eta_{u} I_{u}+\eta_{n} I_{n}-\left(\sigma_{h}+\mu+\delta_{h}\right) I_{h} \\
\frac{\mathrm{d} R}{\mathrm{~d} t}=\sigma_{u} I_{u}+\sigma_{n} I_{n}+\sigma_{h} I_{h}-(\theta+\mu) R \\
\frac{\mathrm{d} V}{\mathrm{~d} t}=\alpha_{u} I_{u}+\alpha_{n} I_{n}-\mu_{c} V
\end{array}\right.
$$

All the parameters involved in the above system are positive. We present the parameters with their biological interpretation, dimensions, and realistic values in Table 1.

\section{Mathematical analysis}

\subsection{Basic properties}

Here, we explore the basic dynamical properties of the model (1). Let us first consider the initial conditions of the model as

$S(0)>0, E(0), I_{u}(0), I_{n}(0), I_{h}(0), R(0), V(0) \geq 0$.

The dynamical system (1) is well posed as shown in the following theorem.

Theorem 1 Consider the model (1) with initial conditions (2). The nonnegative orthant $\mathbb{R}_{+}^{7}$ is invariant under the flow of (1), including $S$ remaining positive with the advancement of time. Moreover, the solutions of the system (1) are bounded in the region

$$
\begin{aligned}
\Omega= & \left\{\left(S, E, I_{u}, I_{n}, I_{h}, R, V\right) \mid 0<S+E+I_{u}+I_{n}\right. \\
& \left.+I_{h}+R \leq \frac{\Pi}{\mu}, \quad 0 \leq V<\frac{\Pi\left(\alpha_{u}+\alpha_{n}\right)}{\mu \mu_{c}}\right\} .
\end{aligned}
$$

Proof Consider the initial conditions (2). Suppose that at time $t=t_{1}, S\left(t_{1}\right)=0$. Then from (1) at $t=t_{1}$, $\frac{\mathrm{d} S}{\mathrm{~d} t}=\Pi+\theta R>0$ which implies that $\frac{\mathrm{d} S}{\mathrm{~d} t}>0$ when $S$ is positive and small. Thus, there is no time $t_{1}$ such that $S\left(t_{1}\right)=0$. Therefore, $S$ stays positive for $t>0$ with the initial condition $S(0)>0$. Now, for the other components

$$
\begin{aligned}
& \left.\frac{\mathrm{d} E}{\mathrm{~d} t}\right|_{E=0}=\frac{\beta_{1} S\left(I_{u}+\nu I_{n}\right)}{N}+\beta_{2} S V \geq 0, \\
& \left.\frac{\mathrm{d} I_{u}}{\mathrm{~d} t}\right|_{I_{u}=0}=(1-p) \gamma E \geq 0, \\
& \left.\frac{\mathrm{d} I_{n}}{\mathrm{~d} t}\right|_{I_{n}=0}=p \gamma E \geq 0, \\
& \left.\frac{\mathrm{d} I_{h}}{\mathrm{~d} t}\right|_{I_{h}=0}=\eta_{u} I_{u}+\eta_{n} I_{n}, \geq 0, \\
& \left.\frac{\mathrm{d} R}{\mathrm{~d} t}\right|_{R=0}=\sigma_{u} I_{u}+\sigma_{n} I_{n}+\sigma_{h} I_{h}, \geq 0, \\
& \left.\frac{\mathrm{d} V}{\mathrm{~d} t}\right|_{V=0}=\alpha_{u} I_{u}+\alpha_{n} I_{n} \geq 0 .
\end{aligned}
$$

Thus, all the other components are nonnegative and the orthant $\mathbb{R}_{+}^{7}$ is invariant under (1).

To show the boundedness of solutions of the system (1), we add all the equations except the last one of (1) to get

$$
\begin{aligned}
\frac{\mathrm{d} N}{\mathrm{~d} t} & =\frac{\mathrm{d}}{\mathrm{d} t}\left(S+E+I_{u}+I_{n}+I_{h}+R\right) \\
& =\Pi-\mu N-\delta_{n} I_{n}-\delta_{h} I_{h} \\
& \leq \Pi-\mu N .
\end{aligned}
$$


Table 1 Description of model parameters used in the model (1)

\begin{tabular}{|c|c|c|c|c|}
\hline Parameter & Dimension & Interpretation & Value(s) & References \\
\hline$\Pi$ & person day ${ }^{-1}$ & $\begin{array}{l}\text { Recruitment rate of suscepti- } \\
\text { bles }\end{array}$ & $\mu \times N_{i n i t}$ & - \\
\hline$\beta_{1}$ & day $^{-1}$ & $\begin{array}{l}\text { Transmission rate for direct } \\
\text { contact }\end{array}$ & $(0,1)$ & To be estimated \\
\hline$\beta_{2}$ & copies $^{-1}$ litre day ${ }^{-1}$ & $\begin{array}{l}\text { Transmission rate for free } \\
\text { virus contact }\end{array}$ & $(0,1)$ & To be estimated \\
\hline$\nu$ & unitless & $\begin{array}{l}\text { Modification factor for noti- } \\
\text { fied infectives }\end{array}$ & 0.1852 & {$[13]$} \\
\hline$\theta$ & day $^{-1}$ & $\begin{array}{l}\text { Rate at which recovered indi- } \\
\text { viduals lose immunity }\end{array}$ & $1 / 365$ & {$[12]$} \\
\hline$\mu$ & day $^{-1}$ & Natural mortality rate & $0.3891 \times 10^{-4}$ & {$[8]$} \\
\hline$\gamma$ & day $^{-1}$ & $\begin{array}{l}\text { Rate at which the exposed } \\
\text { individuals are infected }\end{array}$ & 0.2 & {$[21,22]$} \\
\hline$\eta_{u}$ & day $^{-1}$ & $\begin{array}{l}\text { Rate at which un-notified } \\
\text { patients become hospitalized }\end{array}$ & $(0,1)$ & To be estimated \\
\hline$\eta_{n}$ & day $^{-1}$ & $\begin{array}{l}\text { Rate at which notified } \\
\text { patients become hospitalized }\end{array}$ & $(0,1)$ & To be estimated \\
\hline$p$ & unitless & $\begin{array}{l}\text { Proportion of notified infec- } \\
\text { tives }\end{array}$ & 0.2 & {$[50,52]$} \\
\hline$\sigma_{u}$ & day $^{-1}$ & $\begin{array}{l}\text { Recovery rate of un-notified } \\
\text { patients }\end{array}$ & 0.17 & {$[42,48]$} \\
\hline$\sigma_{n}$ & day $^{-1}$ & $\begin{array}{l}\text { Recovery rate of notified } \\
\text { patients }\end{array}$ & 0.072 & {$[24]$} \\
\hline$\sigma_{h}$ & day $^{-1}$ & $\begin{array}{l}\text { Recovery rate of hospitalized } \\
\text { patients }\end{array}$ & $(0,1)$ & To be estimated \\
\hline$\delta_{n}$ & day $^{-1}$ & $\begin{array}{l}\text { Disease induced mortality } \\
\text { rate of notified patients }\end{array}$ & 0.0017 & {$[13]$} \\
\hline$\delta_{h}$ & day $^{-1}$ & $\begin{array}{l}\text { Disease induced mortality } \\
\text { rate of hospitalized patients }\end{array}$ & $(0,1)$ & To be estimated \\
\hline$\alpha_{u}$ & copies litre $^{-1}$ person $^{-1}$ day $^{-1}$ & $\begin{array}{l}\text { Virus shedding rate of un- } \\
\text { notified patients }\end{array}$ & $(0,10)$ & To be estimated \\
\hline$\alpha_{n}$ & copies litre ${ }^{-1}$ person $^{-1}$ day $^{-1}$ & $\begin{array}{l}\text { Virus shedding rate of notified } \\
\text { patients }\end{array}$ & $(0,10)$ & To be estimated \\
\hline$\mu_{c}$ & day $^{-1}$ & $\begin{array}{l}\text { Natural clearance rate of free } \\
\text { virus }\end{array}$ & 1 & {$[32]$} \\
\hline
\end{tabular}

Using the results of differential inequality, we have

$$
\limsup _{t \rightarrow \infty} N(t) \leq \frac{\Pi}{\mu}
$$

Since the total population is bounded, so the individual components are also bounded.

Also, from the last equation of (1), the rate of change of free virus is given by

$$
\begin{aligned}
\frac{\mathrm{d} V}{\mathrm{~d} t} & =\alpha_{u} I_{u}+\alpha_{n} I_{n}-\mu_{c} V \\
& <\left(\alpha_{u}+\alpha_{n}\right) \frac{\Pi}{\mu}-\mu_{c} V
\end{aligned}
$$

Again, using the results of differential inequality, we have

$$
\limsup _{t \rightarrow \infty} V(t)<\frac{\Pi\left(\alpha_{u}+\alpha_{n}\right)}{\mu \mu_{c}} .
$$

This completes the proof.

\subsection{Disease-free equilibrium and basic reproduction number}

As of many epidemiological models, our model (1) also exhibits the disease-free equilibrium (DFE). At this equilibrium, the population persists in the absence of disease. Mathematically, DFE is obtained by assuming all the infected compartments to be zero, i.e., $E, I_{u}, I_{n}, I_{h}$, and $V$ to be zero. Then from the model, $S=\frac{\Pi}{\mu}$ and $R=0$. Thus, the DFE is given by $\mathscr{E}_{s}=\left(\frac{\Pi}{\mu}, 0,0,0,0,0,0\right)$.

The basic reproduction number $\mathscr{R}_{0}$ is interpreted as the average number of secondary cases produced by one infected individual introduced into a population of susceptible individuals. This dimensionless number is a measure of the potential for disease outbreaks. Now to obtain $\mathscr{R}_{0}$, we use next-generation operator method $[9,43]$. 
First we assemble the compartments which are infected (i.e., $E, I_{u}, I_{n}, I_{h}$, and $V$ ) and decomposing the right hand side of the system (1) as $\mathscr{F}-\mathscr{W}$, where $\mathscr{F}$ is the transmission part, expressing the production of new infection, and the transition part is $\mathscr{W}$, which describe the rate of transfer of individuals from one compartment to another. Then we have for the model (1)

$$
\begin{aligned}
& \mathscr{F}=\left(\begin{array}{c}
\frac{\beta_{1} S\left(I_{u}+\nu I_{n}\right)}{N}+\beta_{2} S V \\
0 \\
0 \\
0 \\
0
\end{array}\right) \text { and } \\
& \mathscr{W}=\left(\begin{array}{c}
(\gamma+\mu) E \\
-(1-p) \gamma E+\phi_{u} I_{u} \\
-p \gamma E+\phi_{n} I_{n} \\
-\eta_{u} I_{u}-\eta_{n} I_{n}+\phi_{h} I_{h} \\
-\alpha_{u} I_{u}-\alpha_{n} I_{n}+\mu_{c} V
\end{array}\right),
\end{aligned}
$$

with $\phi_{u}=\eta_{u}+\sigma_{u}+\mu, \phi_{n}=\eta_{n}+\sigma_{n}+\mu+\delta_{n}$, and $\phi_{h}=\sigma_{h}+\mu+\delta_{h}$.

Consider $X=\left(E, I_{u}, I_{n}, I_{h}, V\right)$. Then the derivatives of $\mathscr{F}$ and $\mathscr{W}$ at the DFE $\mathscr{E}_{s}$ are given by

$$
\begin{aligned}
& F=\frac{\partial \mathscr{F}}{\partial X}=\left(\begin{array}{lllll}
0 & \beta_{1} & \beta_{1} \nu & 0 & \frac{\beta_{2} \Pi}{\mu} \\
0 & 0 & 0 & 0 & 0 \\
0 & 0 & 0 & 0 & 0 \\
0 & 0 & 0 & 0 & 0 \\
0 & 0 & 0 & 0 & 0
\end{array}\right) \text { and } \\
& W=\frac{\partial \mathscr{W}}{\partial X}=\left(\begin{array}{ccccc}
(\gamma+\mu) & 0 & 0 & 0 & 0 \\
-(1-p) \gamma & \phi_{u} & 0 & 0 & 0 \\
-p \gamma & 0 & \phi_{n} & 0 & 0 \\
0 & -\eta_{u} & -\eta_{n} & \phi_{h} & 0 \\
0 & -\alpha_{u} & -\alpha_{n} & 0 & \mu_{c}
\end{array}\right) .
\end{aligned}
$$

Now, we calculate the next-generation matrix $F W^{-1}$, whose $(i, j)$-th entry describes the expected number of new infections in compartment $i$ produced by the infected individual originally introduced into compartment $j$. According to Diekmann et al. [9], the basic reproduction number is given by $\mathscr{R}_{0}=\rho\left(F W^{-1}\right)$, where $\rho$ is the spectral radius of the next-generation matrix $F W^{-1}$. A simple calculation leads to the following expression for $\mathscr{R}_{0}$ :

$$
\begin{aligned}
\mathscr{R}_{0}= & \frac{(1-p) \gamma \beta_{1}}{\phi_{u}(\gamma+\mu)}+\frac{p \gamma \nu \beta_{1}}{\phi_{n}(\gamma+\mu)}+\frac{(1-p) \gamma \alpha_{u} \beta_{2}(\Pi / \mu)}{\mu_{c} \phi_{u}(\gamma+\mu)} \\
& +\frac{p \gamma \alpha_{n} \beta_{2}(\Pi / \mu)}{\mu_{c} \phi_{n}(\gamma+\mu)} .
\end{aligned}
$$

Epidemiologically, the first and second terms of $\mathscr{R}_{0}$ can be interpreted as the number of secondary infections that one un-notified and notified individual will produce in a completely susceptible population during its infectious period, respectively. Here, the infection occurs due to direct contact. Also, the last two terms represent the same but for free virus contact. Thus, both transmission pathways have impacts on the dynamics of COVID-19 spread in the community.

\subsection{Stability of disease-free equilibrium}

Following theorem gives the local stability properties of the DFE $\mathscr{E}_{s}=\left(\frac{\Pi}{\mu}, 0,0,0,0,0,0\right)$.

Theorem 2 The disease-free equilibrium (DFE) $\mathscr{E}_{S}=$ $\left(\frac{\Pi}{\mu}, 0,0,0,0,0,0\right)$ of the system (1) is locally asymptotically stable if $\mathscr{R}_{0}<1$ and unstable if $\mathscr{R}_{0}>1$.

Proof The Jacobian matrix at the DFE $\mathscr{E}_{S}$ is given by

$$
J_{\mathscr{\delta}_{s}}=\left[\begin{array}{ccccccc}
-\mu & 0 & -\beta_{1} & -\nu \beta_{1} & 0 & \theta & -\frac{\Pi \beta_{2}}{\mu} \\
0 & -(\gamma+\mu) & \beta_{1} & \nu \beta_{1} & 0 & 0 & \frac{\Pi \beta_{2}}{\mu} \\
0 & (1-p) \gamma & -\phi_{u} & 0 & 0 & 0 & 0 \\
0 & p \gamma & 0 & -\phi_{n} & 0 & 0 & 0 \\
0 & 0 & \eta_{u} & \eta_{n} & -\phi_{h} & 0 & 0 \\
0 & 0 & \sigma_{u} & \sigma_{n} & \sigma_{h} & -(\theta+\mu) & 0 \\
0 & 0 & \alpha_{u} & \alpha_{n} & 0 & 0 & -\mu_{c}
\end{array}\right]
$$

with $\phi_{u}=\eta_{u}+\sigma_{u}+\mu, \phi_{n}=\eta_{n}+\sigma_{n}+\mu+\delta_{n}$, and $\phi_{h}=\eta_{h}+\mu+\delta_{h}$.

The above Jacobian matrix possess three obvious eigenvalues $-\mu,-(\theta+\mu)$ and $-\phi_{h}=-\left(\sigma_{h}+\mu+\delta_{h}\right)$, which are all negative. Also, the remaining eigenvalues $(\lambda)$ are the roots of the equation $\operatorname{det}(M-\lambda I)=0$, where $I$ is the $4 \times 4$ identity matrix and

$$
M=\left[\begin{array}{cccc}
-(\gamma+\mu) & \beta_{1} & \nu \beta_{1} & \frac{\Pi \beta_{2}}{\mu} \\
(1-p) \gamma & -\phi_{u} & 0 & 0 \\
p \gamma & 0 & -\phi_{n} & 0 \\
0 & \alpha_{u} & \alpha_{n} & -\mu_{c}
\end{array}\right] .
$$

Now, $\operatorname{det}(M-\lambda I)=0$ gives

$$
\begin{aligned}
& \left(\lambda+\mu_{c}\right)\left(\lambda+\phi_{n}\right)\left(\lambda+\phi_{u}\right)(\lambda+\gamma+\mu) \\
& \quad-(1-p) \gamma \beta_{1}\left(\lambda+\mu_{c}\right)\left(\lambda+\phi_{n}\right) \\
& \quad-p \gamma \nu \beta_{1}\left(\lambda+\mu_{c}\right)\left(\lambda+\phi_{u}\right) \\
& \quad-(1-p) \gamma \alpha_{u} \frac{\Pi \beta_{2}}{\mu}\left(\lambda+\phi_{n}\right)-\frac{\Pi \beta_{2}}{\mu} p \gamma \alpha_{n}\left(\lambda+\phi_{u}\right)=0 .
\end{aligned}
$$


The above equation can be written as $H(\lambda)=1$, where

$$
\begin{aligned}
H(\lambda)= & \frac{(1-p) \gamma \beta_{1}}{\left(\lambda+\phi_{u}\right)(\lambda+\gamma+\mu)}+\frac{p \gamma \nu \beta_{1}}{\left(\lambda+\phi_{n}\right)(\lambda+\gamma+\mu)} \\
& +\frac{(1-p) \gamma \alpha_{u} \beta_{2}(\Pi / \mu)}{\left(\lambda+\mu_{c}\right)\left(\lambda+\phi_{u}\right)(\lambda+\gamma+\mu)} \\
& +\frac{p \gamma \alpha_{n} \beta_{2}(\Pi / \mu)}{\left(\lambda+\mu_{c}\right)\left(\lambda+\phi_{n}\right)(\lambda+\gamma+\mu)} .
\end{aligned}
$$

We also rewrite $H(\lambda)$ as

$$
H(\lambda)=H_{1}(\lambda)+H_{2}(\lambda)+H_{3}(\lambda)+H_{4}(\lambda),
$$

where $H_{j}(\lambda), j=1,2,3,4$, are the respective term of (5). Now, if $\operatorname{Re}(\lambda) \geq 0$ with $\lambda=x+i y$, then

$$
\left|H_{1}(\lambda)\right|=\frac{(1-p) \gamma \beta_{1}}{\left|\lambda+\phi_{u}\right||\lambda+\gamma+\mu|} \leq H_{1}(x) \leq H_{1}(0) .
$$

Analogously,

$$
\left|H_{j}(\lambda)\right| \leq H_{j}(x) \leq H_{j}(0), \quad \text { for } j=2,3,4 .
$$

Then

$$
\begin{aligned}
|H(\lambda)| & \leq\left|H_{1}(\lambda)\right|+\left|H_{2}(\lambda)\right|+\left|H_{3}(\lambda)\right|+\left|H_{4}(\lambda)\right| \\
& \leq H_{1}(0)+H_{2}(0)+H_{3}(0)+H_{4}(0) \\
& =H(0) .
\end{aligned}
$$

From (3) and (5), we have $H(0)=\mathscr{R}_{0}$. Then $\mathscr{R}_{0}<1$ $\Longrightarrow|H(\lambda)|<1$, which implies there does not exist any solutions of $H(\lambda)=1$ with $\operatorname{Re}(\lambda) \geq 0$.

Therefore if $\mathscr{R}_{0}<1$, all the eigenvalues of $H(\lambda)=1$ have negative real parts and hence the DFE $\mathscr{E}_{S}$ of the system (1) is locally asymptotically stable.

Now for the case of $\mathscr{R}_{0}>1$, i.e., $H(0)>1$

$$
\lim _{\lambda \rightarrow \infty} H(\lambda)=0 .
$$

Then there exists $\lambda^{*}>0$ such that $H\left(\lambda^{*}\right)=1$, which confirm the existence of positive eigenvalue of the Jacobian matrix $J_{\mathscr{E}_{s}}$. Thus the DFE $\mathscr{E}_{s}$ is unstable for $\mathscr{R}_{0}>1$.

Moreover, when $\mathscr{R}_{0}<1$ the DFE $\mathscr{E}_{s}$ is globally asymptotically stable, which can be assured by the following theorem.

Theorem 3 The disease-free equilibrium $(D F E) \mathscr{E}_{s}=$ $\left(\frac{\Pi}{\mu}, 0,0,0,0,0,0\right)$ of the system (1) is globally asymptotically stable if $\mathscr{R}_{0}<1$.

Proof First we rewrite the model (1) into the form

$$
\left\{\begin{array}{l}
\frac{\mathrm{d} \mathbb{X}}{\mathrm{d} t}=P(\mathbb{X}, \mathbb{Z}), \\
\frac{\mathrm{d} \mathbb{Z}}{\mathrm{d} t}=Q(\mathbb{X}, \mathbb{Z})
\end{array}\right.
$$

where $\mathbb{X}^{\mathrm{T}}=(S, R) \in \mathbb{R}_{+}^{2}$ with $S>0$, describe the uninfected compartments and $\mathbb{Z}^{\mathrm{T}}=\left(E, I_{u}, I_{n}, I_{h}, V\right) \in$ $\mathbb{R}_{+}^{5}$ describe the infected compartments with free virus. Also, $T$ denoting the transpose of the matrix. Note that $Q(\mathbb{X}, \mathbf{0})=\mathbf{0}$, where $\mathbf{0}$ is a zero vector.

Following Castillo-Chavez et al. [4], the DFE $\mathscr{E}_{s}$ of the system (6) is globally asymptotically stable if it is locally asymptotically stable and satisfy following two conditions:

(H1) For the subsystem $\frac{d \mathbb{X}}{d t}=P(\mathbb{X}, \mathbf{0})$, the equilibrium $\mathbb{X}^{*}$ is globally asymptotically stable, $Q(\mathbb{X}, \mathbb{Z})=B \mathbb{Z}-\widetilde{Q}(\mathbb{X}, \mathbb{Z}), \widetilde{Q}(\mathbb{X}, \mathbb{Z}) \geq 0$ for $(\mathbb{X}, \mathbb{Z}) \in$ $\Omega$,

where $B=\left.\frac{\partial Q}{\partial \mathbb{Z}}\right|_{(\mathbb{X}, \mathbf{Z})=\left(\mathbb{X}^{*}, \mathbf{0}\right)}$ is a Metzler matrix (a matrix whose off diagonal elements are nonnegative) and $\Omega$ is the positive invariant set for the model (1) as described in Sect. 3.1.

Now, for the model (1),

$$
P(\mathbb{X}, \mathbf{0})=\left(\begin{array}{c}
\Pi-\mu S \\
0
\end{array}\right) .
$$

Since all the infected compartments are zero, so there is no infection, and thus, no recovery. For this reason, we consider $R=0$. Clearly, $\mathbb{X}^{*}=\left(\frac{\Pi}{\mu}, 0\right)$ is a globally asymptotically equilibrium for the system $\frac{\mathrm{dX}}{\mathrm{d} t}=$ $P(\mathbb{X}, \mathbf{0})$. So the condition (H1) is satisfied. Now,

$$
\begin{aligned}
B & =\left(\begin{array}{ccccc}
-(\gamma+\mu) & \beta_{1} & \beta_{1} \nu & 0 & \frac{\beta_{2} \Pi}{\mu} \\
(1-p) \gamma & -\phi_{u} & 0 & 0 & 0 \\
p \gamma & 0 & -\phi_{n} & 0 & 0 \\
0 & \eta_{u} & \eta_{n} & -\phi_{h} & 0 \\
0 & \alpha_{u} & \alpha_{n} & 0 & -\mu_{c}
\end{array}\right) \text { and } \\
\widetilde{Q}(\mathbb{X}, \mathbb{Z}) & =\left(\begin{array}{c}
\beta_{1}\left(1-\frac{S}{N}\right) I_{u}+\beta_{1} \nu \\
\left(1-\frac{S}{N}\right) I_{n}+\beta_{2}\left(\frac{\Pi}{\mu}-S\right) V \\
0 \\
0 \\
0 \\
0
\end{array}\right),
\end{aligned}
$$

with $\phi_{u}=\eta_{u}+\sigma_{u}+\mu, \phi_{n}=\eta_{n}+\sigma_{n}+\mu+\delta_{n}$, and $\phi_{h}=\eta_{h}+\mu+\delta_{h}$.

The matrix $B$ is a Metzler matrix and $\widetilde{Q}(\mathbb{X}, \mathbb{Z}) \geq 0$ whenever the state variables are inside the invariant set $\Omega$. Thus (H2) is also satisfied. This completes the proof.

\subsection{Existence of endemic equilibrium}

Here, we discuss the existence of endemic equilibrium of the model (1). The endemic equilibrium $\mathscr{E}^{*}=$ $\left(S^{*}, E^{*}, I_{u}^{*}, I_{n}^{*}, I_{h}^{*}, R^{*}, V^{*}\right)$ is given by $I_{u}^{*}=A_{1} E^{*}$, $I_{n}=A_{2} E^{*}, I_{h}^{*}=A_{3} E^{*}, R=A_{4} E^{*}, V^{*}=A_{5} E^{*}$, 
$S^{*}=\frac{\Pi+\left(\theta A_{4}-(\gamma+\mu)\right) E^{*}}{\mu}=\frac{(\gamma+\mu)\left(\Pi-A_{7} E^{*}\right)}{A_{6}+\beta_{2} A_{5}\left(\Pi-A_{7} E^{*}\right)}$, where all the $A_{i}, i=1,2, \ldots, 7$, are positive and given by

$$
\begin{aligned}
& A_{1}=\frac{(1-p) \gamma}{\eta_{u}+\sigma_{u}+\mu}, \quad A_{2}=\frac{p \gamma}{\eta_{n}+\sigma_{n}+\mu+\delta_{n}}, \\
& A_{3}=\frac{\eta_{u} A_{1}+\eta_{n} A_{2}}{\sigma_{h}+\mu+\delta_{h}}, \\
& A_{4}=\frac{\sigma_{u} A_{1}+\sigma_{n} A_{2}+\sigma_{h} A_{3}}{\theta+\mu}, \quad A_{5}=\frac{\alpha_{u} A_{1}+\alpha_{n} A_{2}}{\mu_{c}}, \\
& A_{6}=\mu \beta_{1}\left(A_{1}+\nu A_{2}\right), \\
& A_{7}=\delta_{n} A_{2}+\delta_{h} A_{3},
\end{aligned}
$$

and $E^{*}$ is a positive root of the quadratic equation

$$
\rho_{0} E^{2}+\rho_{1} E+\rho_{2}=0
$$

with

$$
\begin{aligned}
\rho_{0}= & -\beta_{2} A_{5} A_{7}\left(\theta A_{4}-(\gamma+\mu)\right), \\
\rho_{1}= & \left(A_{6}+\beta_{2} A_{5} \Pi\right)\left(\theta A_{4}-(\gamma+\mu)\right)-\beta_{2} A_{5} A_{7} \Pi \\
& +\mu(\gamma+\mu) A_{7} \\
= & \mu(\gamma+\mu) \mathscr{R}_{0}\left(\theta A_{4}-(\gamma+\mu)\right)+\mu(\gamma+\mu)\left(1-\mathscr{R}_{0}\right) A_{7} \\
& +A_{6} A_{7}, \\
\rho_{2}= & \left(A_{6}+\beta_{2} A_{5} \Pi\right) \Pi-\mu(\gamma+\mu) \Pi=\mu(\gamma+\mu) \Pi\left(\mathscr{R}_{0}-1\right) .
\end{aligned}
$$

For the existence of real roots of (7), we must have $\rho_{1}^{2}-4 \rho_{0} \rho_{2} \geq 0$. Without loss of generality, assume that $E_{1}^{*}>E_{2}^{*}$, when Eq. (7) have two real roots. From the expression of $S^{*}, S^{*}=\frac{\Pi}{\mu}+\frac{\left(\theta A_{4}-(\gamma+\mu)\right)}{\mu} E^{*}$. Since the upper bound of total population is $\Pi / \mu$, so we must have $\theta A_{4}-(\gamma+\mu)<0$. Then $\rho_{0}$ is always positive. Note that, for $\theta A_{4}-(\gamma+\mu)=0, S^{*}=\frac{\Pi}{\mu}$ and thus the endemic equilibrium becomes DFE. Also, $\rho_{2}<(>) 0 \Longleftrightarrow \mathscr{R}_{0}<$ $(>) 1$. At the equilibrium density, $N^{*}=\frac{\Pi-A_{7} E^{*}}{\mu}$. Thus, $N^{*}>0 \Longleftrightarrow \Pi-A_{7} E^{*}>0$. Keeping all the conditions in mind, in the next theorem, we state the existence and the possible number of endemic equilibrium points.

Theorem 4 When $\mathscr{R}_{0}<1$, the model (1) has unique endemic equilibrium if $\Pi-A_{7} E^{*}>0$. When $\mathscr{R}_{0}>1$, the model (1) has

1. two endemic equilibrium if $\rho_{1}<0, \rho_{1}^{2}-4 \rho_{0} \rho_{2}>0$, and $\Pi-A_{7} E_{i}^{*}>0, i=1,2$.

2. unique endemic equilibrium if any of the following cases holds
(a) $\rho_{1}<0, \rho_{1}^{2}-4 \rho_{0} \rho_{2}>0$, and $E_{1}^{*}>\frac{\Pi}{A_{7}}>E_{2}^{*}$,
(b) $\rho_{1}<0, \rho_{1}^{2}-4 \rho_{0} \rho_{2}=0, \Pi-A_{7} E^{*}>0$,

3. no endemic equilibrium otherwise.

\subsection{Analysis of the center manifold near DFE $\mathscr{E}_{s}$ when $\mathscr{R}_{0}=1$}

In this subsection, we discuss the nature of DFE $\mathscr{E}_{s}$ when $\mathscr{R}_{0}=1$. Recall the Jacobian matrix $J_{\mathscr{E}_{s}}$ from $(4)$.
Notice that, when $\mathscr{R}_{0}=1, J_{\mathscr{E}}$ possess a zero eigenvalue. So the equilibrium $\mathscr{E}_{s}$ becomes non-hyperbolic. Then the center manifold theory is the best approach to study the behaviour of this equilibrium. In this regard, we follow Theorem 4.1 described in Castillo-Chavez and Song [5].

To proceed, first we write the mathematical model (1) into the vector form $\frac{\mathrm{d} \mathbf{x}}{\mathrm{d} t}=f(\mathbf{x})$, with $\mathbf{x}=$ $\left(x_{1}, x_{2}, x_{3}, x_{4}, x_{5}, x_{6}, x_{7}\right)^{\mathrm{T}}=\left(S, E, I_{u}, I_{n}, I_{h}, R\right.$, $V)^{\mathrm{T}}$ and $f(\mathbf{x})=\left(f_{1}(\mathbf{x}), f_{2}(\mathbf{x}), \ldots, f_{7}(\mathbf{x})\right)^{\mathrm{T}}$. Since $\mathscr{R}_{0}$ is often inconvenient to use directly as a bifurcation parameter, we introduce $\beta_{1}$ as a bifurcation parameter. Then $\mathscr{R}_{0}=1$ gives

$$
\begin{aligned}
\beta_{1} & =\frac{\mu_{c}(\gamma+\mu) \phi_{u} \phi_{n}-\gamma \beta_{2}(\Pi / \mu)\left((1-p) \alpha_{u} \phi_{n}+p \alpha_{n} \phi_{u}\right)}{\mu_{c} \gamma\left((1-p) \phi_{n}+\nu p \phi_{u}\right)} \\
& =\beta_{1}^{*} \text { (say). }
\end{aligned}
$$

Moreover, $\mathscr{R}_{0}<1$ for $\beta_{1}<\beta_{1}^{*}$ and $\mathscr{R}_{0}>1$ for $\beta_{1}>\beta_{1}^{*}$. Now, consider the system $\frac{\mathrm{d} \mathbf{x}}{\mathrm{d} t}=f\left(\mathbf{x}, \beta_{1}\right)$ and at $\beta_{1}=\beta_{1}^{*}$ the Jacobian matrix $J_{\mathscr{E}_{s}}$ have simple zero eigenvalue and all the other eigenvalues have negative real parts. Let $\mathbf{v}=\left(v_{1}, v_{2}, \ldots, v_{7}\right)$ and $\mathbf{w}=\left(w_{1}, w_{2}, \ldots, w_{7}\right)^{\mathrm{T}}$ are the respective left and right eigenvectors corresponding to zero eigenvalue. Simple algebraic calculations lead us to the following expressions of $v_{i}$ and $w_{i}$, $i=1,2, \ldots, 7$ :

$$
\begin{gathered}
v_{1}=0=v_{5}=v_{6}, \quad v_{2}=v_{2}>0, \\
v_{3}=\frac{1}{\phi_{u}}\left(\beta_{1}^{*}+\frac{\beta_{2} \alpha_{u} \Pi}{\mu \mu_{c}}\right) v_{2}, \\
v_{4}=\frac{1}{\phi_{n}}\left(\beta_{1}^{*} \nu+\frac{\beta_{2} \alpha_{n} \Pi}{\mu \mu_{c}}\right) v_{2}, \\
v_{7}=\frac{\beta_{2} \Pi}{\mu \mu_{c}} v_{2},
\end{gathered}
$$

and

$$
\begin{aligned}
w_{1}= & \frac{1}{\mu}\left[\frac { \theta } { \theta + \mu } \left\{\frac{(1-p) \gamma}{\phi_{u}}\left(\sigma_{u}+\frac{\sigma_{h} \eta_{u}}{\phi_{h}}\right)\right.\right. \\
& \left.\left.+\frac{p \gamma}{\phi_{n}}\left(\sigma_{n}+\frac{\sigma_{h} \eta_{n}}{\phi_{h}}\right)\right\}-(\gamma+\mu)\right] w_{2}, \\
w_{2}= & w_{2}>0, \quad w_{3}=\frac{(1-p) \gamma}{\phi_{u}} w_{2}, \quad w_{4}=\frac{p \gamma}{\phi_{n}} w_{2}, \\
w_{5}= & \frac{1}{\phi_{h}}\left(\frac{(1-p) \gamma \eta_{u}}{\phi_{u}}+\frac{p \gamma \eta_{n}}{\phi_{n}}\right) w_{2}, \\
w_{6}= & \frac{1}{\theta+\mu}\left[\frac{(1-p) \gamma}{\phi_{u}}\left(\sigma_{u}+\frac{\sigma_{h} \eta_{u}}{\phi_{h}}\right)\right. \\
& \left.+\frac{p \gamma}{\phi_{n}}\left(\sigma_{n}+\frac{\sigma_{h} \eta_{n}}{\phi_{h}}\right)\right] w_{2}, \\
w_{7}= & \frac{1}{\mu_{c}}\left(\frac{(1-p) \gamma \alpha_{u}}{\phi_{u}}+\frac{p \gamma \alpha_{n}}{\phi_{n}}\right) w_{2} .
\end{aligned}
$$

Clearly, all the $v_{i}$ and $w_{i}(i=1,2, \ldots, 7)$ are positive except $w_{1}$. From the expression of $w_{1}$, note that $w_{1}=$ 
$\frac{1}{\mu}\left(\theta A_{4}-(\gamma+\mu)\right) w_{2}$. Also, since $\theta A_{4}-(\gamma+\mu)<0$, so $w_{1}<0$. Now, according to the Theorem 4.1 [5], we calculate the quantities $a$ and $b$, which are defined by

$$
\begin{aligned}
& a=\sum_{k, i, j=1}^{7} v_{k} w_{i} w_{j} \frac{\partial^{2} f_{k}}{\partial x_{i} \partial x_{j}}, \\
& b=\sum_{k, i=1}^{7} v_{k} w_{i} \frac{\partial^{2} f_{k}}{\partial x_{i} \partial \beta_{1}},
\end{aligned}
$$

where all the partial derivatives are evaluated at the DFE $\mathscr{E}_{S}$ and $\beta_{1}=\beta_{1}^{*}$. Since $v_{1}$ is zero and $f_{k}, k=$ $3,4, \ldots 7$ are linear functions of the state variables, so we need to calculate only $\frac{\partial^{2} f_{2}}{\partial x_{i} \partial x_{j}}$. Then we have

$$
\begin{aligned}
& \frac{\partial^{2} f_{2}}{\partial x_{1} \partial x_{7}}=\beta_{2}, \quad \frac{\partial^{2} f_{2}}{\partial x_{2} \partial x_{3}}=-\frac{\beta_{1}^{*} \mu}{\Pi}=\frac{\partial^{2} f_{2}}{\partial x_{3} \partial x_{5}}=\frac{\partial^{2} f_{2}}{\partial x_{3} \partial x_{6}}, \\
& \frac{\partial^{2} f_{2}}{\partial x_{3} \partial x_{4}}=-\frac{\beta_{1}^{*} \mu(1+\nu)}{\Pi}, \\
& \frac{\partial^{2} f_{2}}{\partial x_{3}^{2}}=-\frac{2 \beta_{1}^{*} \mu}{\Pi}, \quad \frac{\partial^{2} f_{2}}{\partial x_{2} \partial x_{4}}=-\frac{\beta_{1}^{*} \mu \nu}{\Pi}=\frac{\partial^{2} f_{2}}{\partial x_{4} \partial x_{5}}=\frac{\partial^{2} f_{2}}{\partial x_{4} \partial x_{6}}, \\
& \frac{\partial^{2} f_{2}}{\partial x_{4}^{2}}=-\frac{2 \beta_{1}^{*} \mu \nu}{\Pi},
\end{aligned}
$$

and the rest of the derivatives are zero. Thus from (8), we have

$$
\begin{aligned}
a= & v_{2}\left[\beta_{2} w_{1} w_{7}-\frac{\beta_{1}^{*} \mu}{\Pi}\left\{w_{3}\left(w_{2}+2 w_{3}+w_{4}+w_{5}+w_{6}\right)\right.\right. \\
& \left.\left.+\nu w_{4}\left(w_{2}+w_{3}+2 w_{4}+w_{5}+w_{6}\right)\right\}\right] .
\end{aligned}
$$

Again, since $v_{1}$ is zero and $\frac{\partial^{2} f_{k}}{\partial x_{i} \partial \beta_{1}}=0$ for $k=$ $3,4, \ldots, 7$, then from (9) we have

$b=v_{2}\left[w_{3} \frac{\partial^{2} f_{2}}{\partial x_{3} \partial \beta_{1}}+w_{4} \frac{\partial^{2} f_{2}}{\partial x_{4} \partial \beta_{1}}\right]=v_{2}\left(w_{3}+\nu w_{4}\right)>0$.

Since $w_{1}<0$, so $a<0$. Hence, for the system (1), $a<0$ and $b>0$. Thus the system exhibit forward bifurcation at $\beta_{1}=\beta_{1}^{*}$, and an endemic equilibrium appears which is locally asymptotically stable.

We numerically verify the existence of forward bifurcation with respect to the basic reproduction number $\mathscr{R}_{0}$. Since $\mathscr{R}_{0}$ is not a model parameter and it depends on $\beta_{1}$, so we vary $\beta_{1}$. When $\mathscr{R}_{0}$ crosses the threshold value 1 , the system transits from the stable DFE to the stable endemic equilibrium and the DFE becomes unstable (Fig. 2). In the figure, the blue-coloured line depicts the stable DFE and the red curve depicts the stable endemic equilibrium.

We also draw the time series plot by choosing two different values of $\beta_{1}$. For $\beta_{1}=0.15, \mathscr{R}_{0}<1$ and all the infected compartments (un-notified, notified and hospitalized) becomes zero and for $\beta_{1}=0.3, \mathscr{R}_{0}>1$ and infected compartments persists in a stable manner as time evolves (Fig. 3).

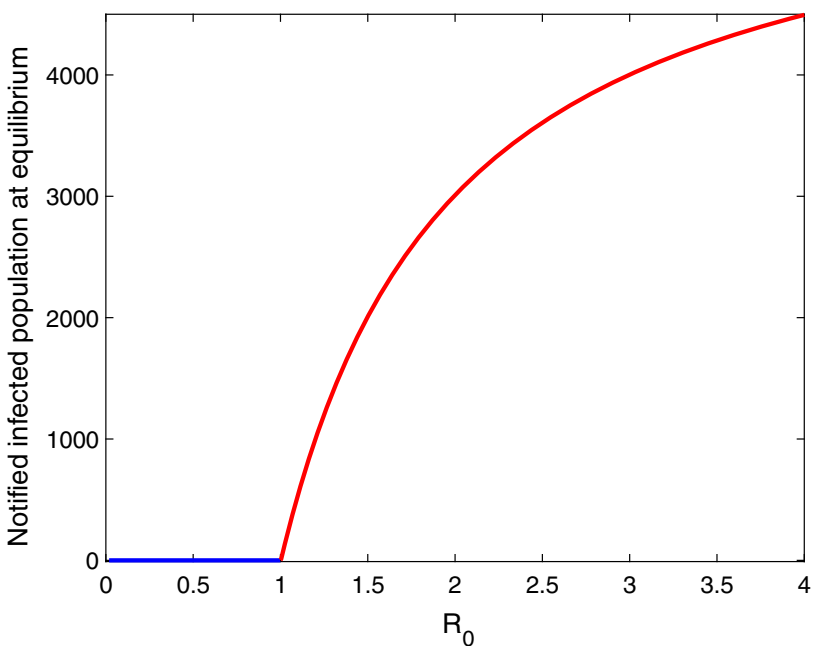

Fig. 2 Forward bifurcation of the system (1) with respect to the basic reproduction number $\mathscr{R}_{0}$. The parameter values are taken as $\Pi=496.68615, \beta_{2}=6.6248 \times 10^{-8}$, $\eta_{u}=0.0223, \eta_{n}=0.7846, \sigma_{h}=0.9181, \delta_{h}=0.0046$, $\alpha_{u}=1.3232 \times 10^{-6}, \alpha_{n}=0.0741$ and the other parameter values are same as Table 1

\section{Model calibration}

Daily new deaths between the period 16 January 2021 (start date of vaccination in India) to 31 October 2021 for the districts Bangalore Urban and Chennai are used to calibrate the model. The rationale behind using mortality data is that this data is more reliable than the incidence data due to limited testing capacities $[20,30]$. We fit the model output $\left(\int_{t=1}^{T}\left[\delta_{n} I_{n}+\delta_{h} I_{h}\right] d t\right.$, where $\mathrm{T}=289)$ to daily new deaths and cumulative deaths due to COVID-19 for both districts. Fixed parameters and initial conditions of the model (1) are given in Tables 1 and 2, respectively. Eight unknown model parameters are estimated, namely $\beta_{1}, \beta_{2}, \nu_{u}$, $\nu_{h}, \sigma_{h}, \delta_{h}, \alpha_{u}$ and $\alpha_{n}$. In addition, the initial number of exposed people $E(0)$ is also estimated from the mortality data. During the specified time period, nonlinear least square solver lsqnonlin (in MATLAB) is used to fit simulated daily mortality data to the reported COVID-19 deaths in Bangalore urban and Chennai districts. MATLAB codes for generating the fitting figures for Chennai have been uploaded to the GitHub repository https://github.com/indrajitg-r/ ODE_model_fitting. The fitting of the daily new deaths and cumulative deaths due to COVID-19 are displayed in Figs. 4 and 5 for Bangalore urban and Chennai, respectively.

From Fig. 4, it can be argued that the model output fits the mortality data well. Estimated parameter values for Bangalore urban are as follows: $\beta_{1}=0.2277$, $\beta_{2}=4.5394 \times 10^{-6}, \eta_{u}=0.0104, \eta_{n}=0.3908, \sigma_{h}=$ $0.7564, \delta_{h}=0.0028, \alpha_{u}=0.0055, \alpha_{n}=4.8891 \times 10^{-6}$ and $E(0)=121$. The data trend is well captured by the model output as seen in Fig. 5. Estimated parameter values for Chennai district data are as follows: 

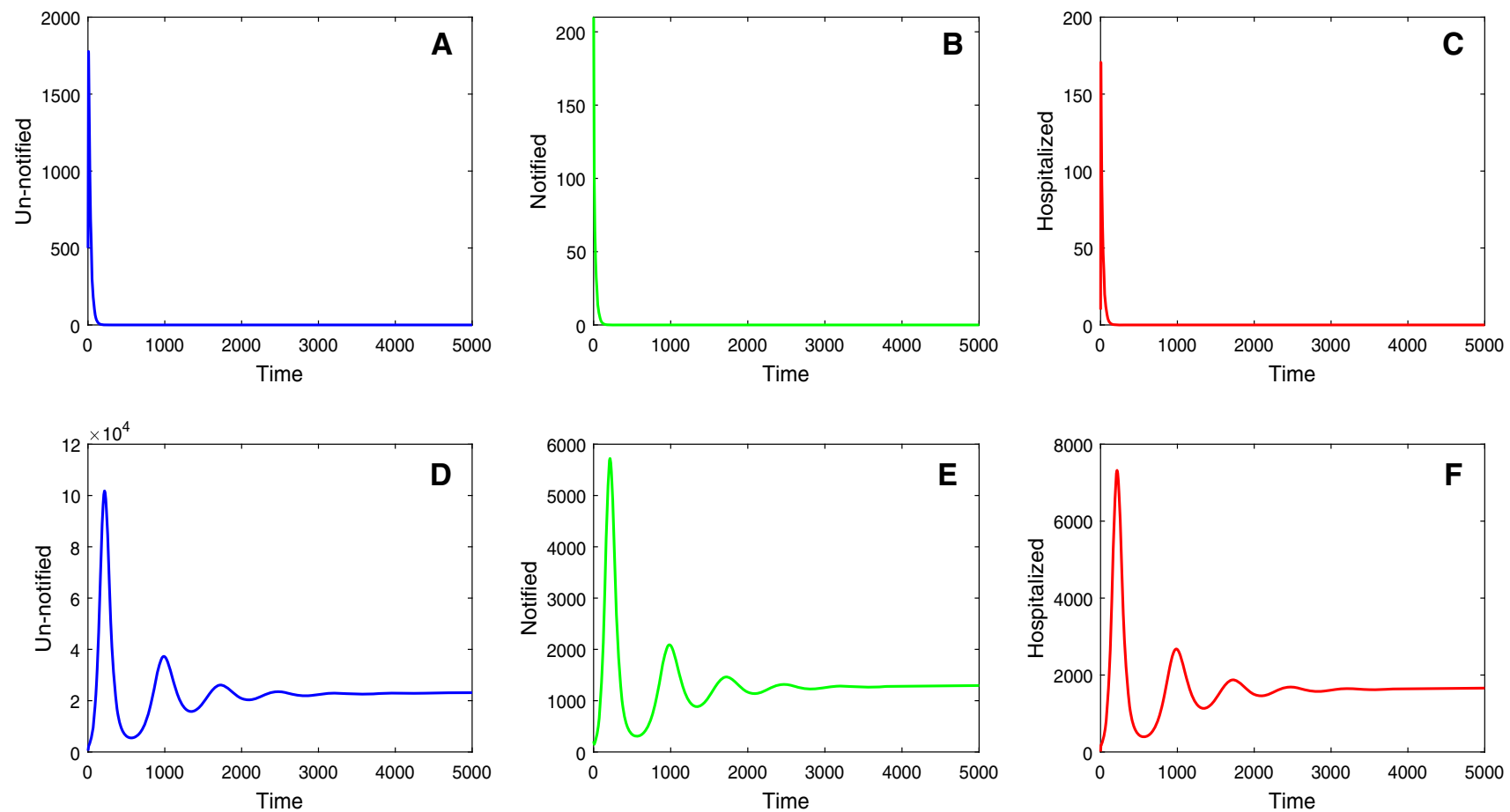

Fig. 3 Time series evaluation of infected compartments for the system (1). In $\mathbf{A}-\mathbf{C}, \beta_{1}=0.15$ where $\mathscr{R}_{0}<1$, and for D-F $\beta_{1}=0.3$ where $\mathscr{R}_{0}>1$. The other parameter values are same as Fig. 2

Table 2 Initial conditions used to simulate the model (1) in Bangalore urban and Chennai

\begin{tabular}{|c|c|c|c|c|}
\hline IC's & Description & $\begin{array}{l}\text { Values for Bangalore } \\
\text { urban }\end{array}$ & Values for Chennai & References \\
\hline$N_{\text {init }}$ & Total population & $12,765,000$ & $11,235,000$ & {$[25,26]$} \\
\hline$S(0)$ & Initial number of susceptible & $0.7 \times N_{\text {init }}$ & $0.7 \times N_{i n i t}$ & - \\
\hline$E(0)$ & $\begin{array}{l}\text { Initial number of exposed peo- } \\
\text { ple }\end{array}$ & $(1-15,000)$ & $(1-15,000)$ & To be estimated \\
\hline$I_{u}(0)$ & $\begin{array}{l}\text { Initial number of un-notified } \\
\text { patients }\end{array}$ & 500 & 500 & - \\
\hline$I_{n}(0)$ & $\begin{array}{l}\text { Initial number of notified } \\
\text { patients }\end{array}$ & 282 & 165 & {$[7]$} \\
\hline$I_{h}(0)$ & $\begin{array}{l}\text { Initial number of hospitalized } \\
\text { patients }\end{array}$ & 10 & 10 & - \\
\hline$R(0)$ & $\begin{array}{l}\text { Initial number of recovered } \\
\text { patients }\end{array}$ & 1000 & 1000 & - \\
\hline$V(0)$ & Initial concentration of virus & $10^{-7}$ & $10^{-7}$ & - \\
\hline
\end{tabular}

$\beta_{1}=0.1962, \beta_{2}=6.4749 \times 10^{-6}, \eta_{u}=2.4095 \times 10^{-7}$, $\eta_{n}=0.9997, \sigma_{h}=0.8244, \delta_{h}=0.0019, \alpha_{u}=0.0011$, $\alpha_{n}=0.0538$ and $E(0)=718$. Using these estimated parameters for both the districts, we investigate different control strategies.

\section{Control interventions and immigration of infectives}

In this section, we investigate different control mechanisms and immigration of infectives through numerical simulation. We examine the effects of vaccination, treatment by drugs, and use of face masks with different degrees of efficacy.

\subsection{Use of face mask}

Although several vaccines are discovered and people are vaccinated in a rapid process, several drugs are in the trial stage and few of them are implemented for hospitalized patients, nevertheless, the use of face mask still could offer as a non-pharmaceutical intervention, to avoid transmission of direct contact and airborne transmission of free virus. In a densely populated country like India, it is almost impossible to determine how many people have come in contact with an infected per- 
(A)

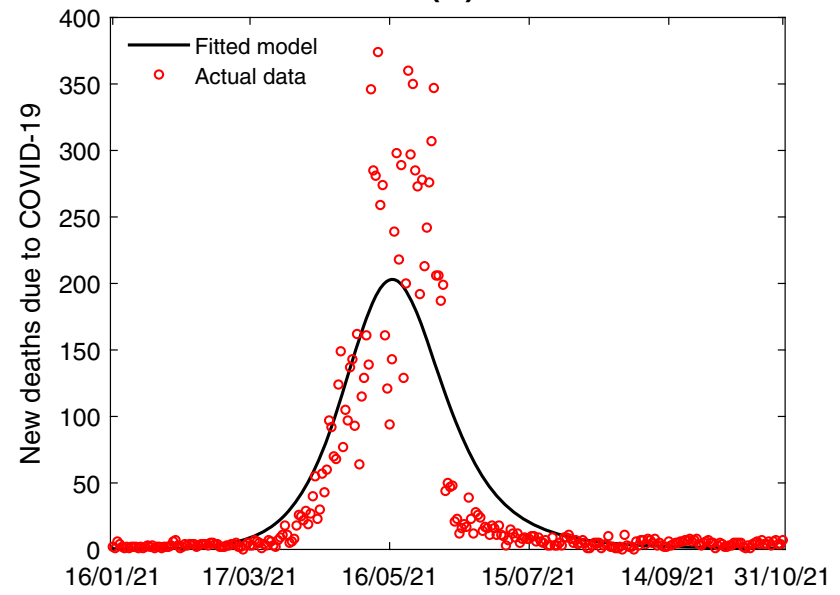

(B)

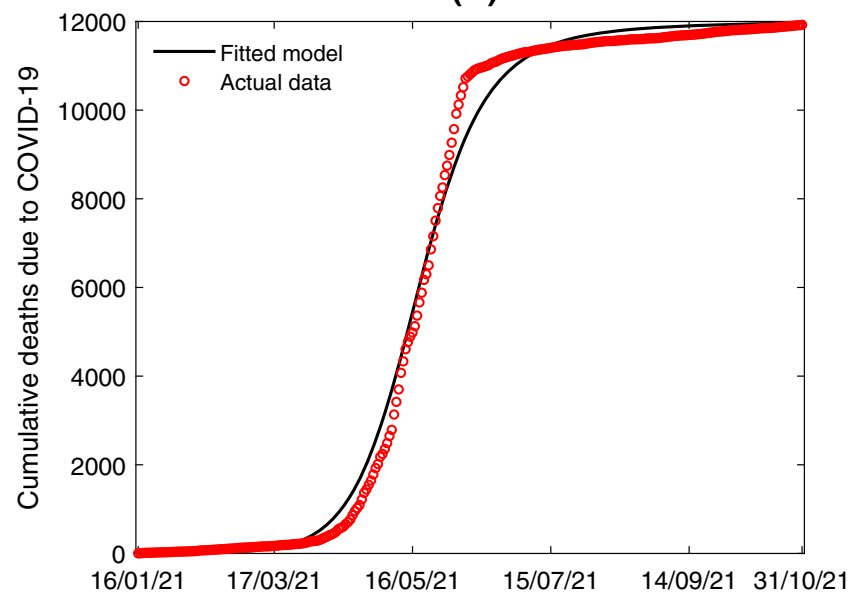

Fig. 4 Fitting model solution to $\mathbf{A}$ new deaths and $\mathbf{B}$ cumulative death data due to COVID-19 in Bangalore urban district

(A)

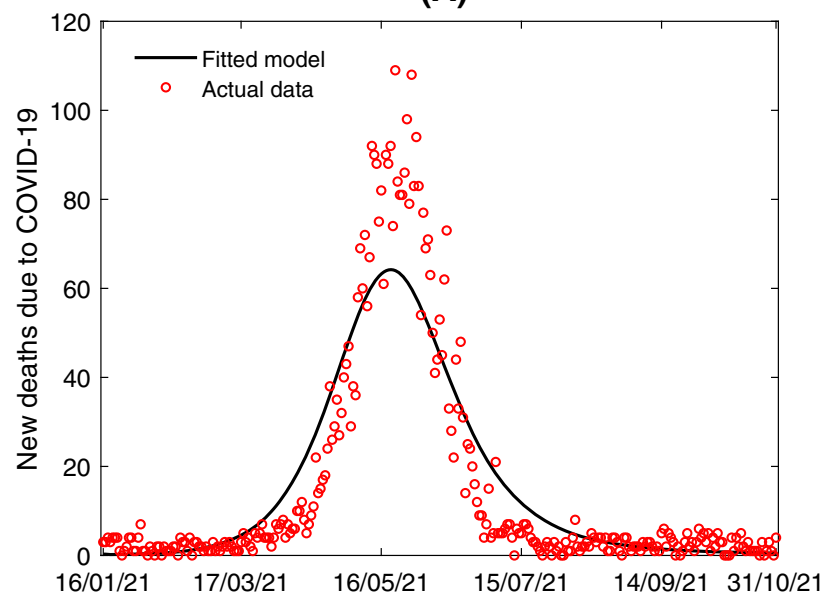

(B)

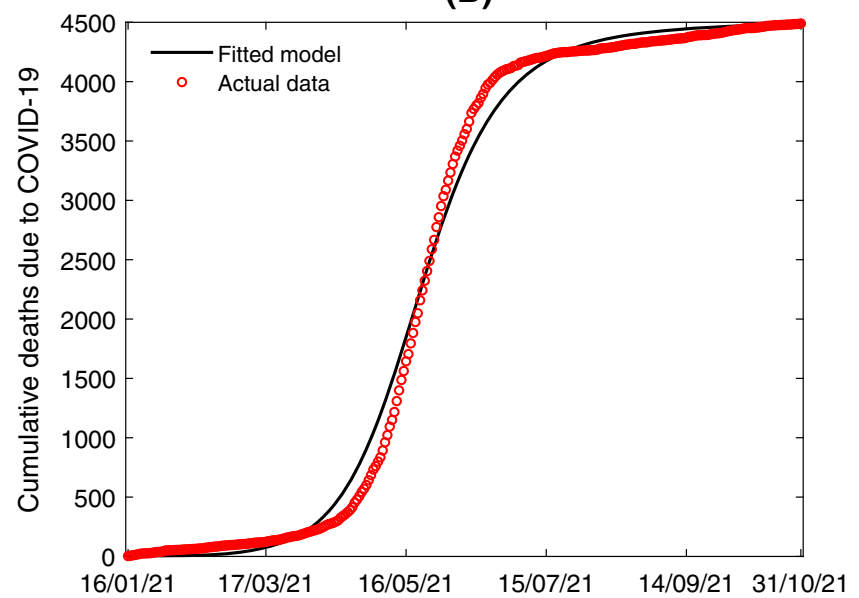

Fig. 5 Fitting model solution to $\mathbf{A}$ new deaths and $\mathbf{B}$ cumulative deaths data due to COVID-19 in Chennai district

son. Thus, wearing a face mask properly is an important control strategy for the current epidemic outbreak. The idea of using a face mask to combat respiratory infections in the community was not new [44]. Face masks reduce the amount of droplet inoculum discharging from infectious individuals by capturing a proportion of droplets within the mask $[40,44]$. Face masks also reduce the amount of droplet inoculum inhaled by susceptible individuals by capturing a proportion of droplets in the inhaled air and hence reducing the airborne transmission rate. Let $0 \leq c_{m} \leq 1$ is the community-wide compliance in the face mask usage and $0<\epsilon_{m} \leq 1$ is the face mask efficiency for preventing the disease. Then the term $1-c_{m} \epsilon_{m}$ describes a measure of reduction in community contacts and free virus contacts due to the use of face mask $[30,40]$. Note that, $0 \leq 1-c_{m} \epsilon_{m} \leq 1$. We consider three different efficiency levels, depending on the material and layering of the face masks viz. N95 masks are 95\% effective; Surgical masks are $55 \%$ effective and Multi-layered cloth masks are $38 \%$ effective [37]. Community-wide compli- ance in the face mask usage is chosen at three different levels namely, High 75\%, Medium 50\%, and low $25 \%$. The baseline values for $c_{m}$ and $\epsilon_{m}$ are taken to be $10 \%$ and 0.1 , respectively.

\subsection{Treatment by drugs: faster recovery}

In recent times, several drugs are developed for moderate to severe COVID-19 patients and some of them are in a different phase of the trial period. The 3rd phase trial of Soin et al. [39] in India predict that tocilizumab plus standard care in patients admitted to hospital with moderate to severe COVID-19 have a faster recovery and reduce the burden of intensive care. Recently, Institute of Nuclear Medicine and Allied Sciences (DRDO-INMAS) and Dr. Reddy's laboratories, Hyderabad jointly developed a drug, 2-deoxy-D-glucose (2-DG) for emergency use in symptomatic COVID-19 patients [3,33]. On 8th May 2021, the Drugs Controller General of India (DCGI) approved this drug [31]. As per 
the government release, clinical trial data shows that the drug helps in the recovery timelines of hospitalized patients and reduces supplemental oxygen dependence. Latterly, pfizer's novel COVID-19 oral antiviral treatment candidate PAXLOVID ${ }^{T M}$ (PF-07321332; ritonavir) is showing significant reduction in deaths or hospitalization in high risk groups. Consider $\epsilon_{t} \geq 1$ be the factor describing the faster recovery of hospitalized patients. Taking the base value of the $\epsilon_{t}$ as 1 , we vary this parameter up to 1.3 while evaluating control strategies.

\subsection{Effect of vaccination}

To control the ongoing global pandemic, vaccine is a critical tool as a pharmaceutical intervention. At present, Covaxin and Covishield vaccines are being used for the vaccination drive against COVID-19 in India and also, several vaccines are in trial process [38]. To extend our mathematical model under the effect of vaccination, we introduced a new compartment of population, namely protected $(P(t))$, those who are vaccinated. Now, the Susceptible and recovered populations both can progress to the protected population at rates $\xi_{s}$ and $\xi_{r}$, respectively, through vaccination. For simplicity, we consider that the protected population completed the required doses of vaccine (and also completed 14 days after the last dose) and thus does not consider any intermediate stage (i.e., the time gap between doses or before 14 days of the last dose). The base values for vaccination rates are chosen to be 0.00085 for both susceptibles and recovered people. This value is calculated from the vaccination data of India [7]. Simulations are performed by increasing the vaccination rates to 0.05 for both sub-populations.

\subsection{Immigration of infectives}

Communicable diseases like the current COVID-19 epidemic may be introduced into a community by the arrival of infectives outside the community. Indeed, in India, the first COVID-19 case was reported in Trissur, Kerala, on January 30, 2020, who was returned from Wuhan, China [2]. Thus, the immigration of infectives in a disease-free community played a crucial role for the spread of the disease. We extend the mathematical model (1) by assuming a constant flow of new members of an un-notified person. Recruitment of notified person was not considered as they are restricted for travelling. On the other hand, immigration is necessary for many people to maintain a livelihood. Thus, individuals from neighbouring locations must be allowed to immigrate for the well-being of the overall community. Let $\Lambda$ be the recruitment rate of un-notified infected individuals through immigration. We consider three levels of immigration as low, medium, and high with $\Lambda=1,5$, and 10 respectively.

By considering the above control interventions and immigration of infectives, the model (1) can be extended to the following model:

$$
\left\{\begin{aligned}
\frac{\mathrm{d} S}{\mathrm{~d} t}= & \Pi-\frac{\beta_{1}\left(1-c_{m} \epsilon_{m}\right) S\left(I_{u}+\nu I_{n}\right)}{N}-\beta_{2}\left(1-c_{m} \epsilon_{m}\right) S V \\
& -\left(\mu+\xi_{s}\right) S+\theta R \\
\frac{\mathrm{d} E}{\mathrm{~d} t}= & \frac{\beta_{1}\left(1-c_{m} \epsilon_{m}\right) S\left(I_{u}+\nu I_{n}\right)}{N}+\beta_{2}\left(1-c_{m} \epsilon_{m}\right) S V-(\gamma+\mu) E \\
\frac{\mathrm{d} I_{u}}{\mathrm{~d} t}= & \Lambda+(1-p) \gamma E-\left(\eta_{u}+\sigma_{u}+\mu\right) I_{u} \\
\frac{\mathrm{d} I_{n}}{\mathrm{~d} t}= & p \gamma E-\left(\eta_{n}+\sigma_{n}+\mu+\delta_{n}\right) I_{n} \\
\frac{\mathrm{d} I_{h}}{\mathrm{~d} t}= & \eta_{u} I_{u}+\eta_{n} I_{n}-\left(\epsilon_{t} \sigma_{h}+\mu+\delta_{h}\right) I_{h} \\
\frac{\mathrm{d} R}{\mathrm{~d} t}= & \sigma_{u} I_{u}+\sigma_{n} I_{n}+\epsilon_{t} \sigma_{h} I_{h}-\left(\theta+\mu+\xi_{r}\right) R \\
\frac{\mathrm{d} P}{\mathrm{~d} t}= & \xi_{s} S+\xi_{r} R-\mu P \\
\frac{\mathrm{d} V}{\mathrm{~d} t}= & \alpha_{u} I_{u}+\alpha_{n} I_{n}-\mu_{c} V
\end{aligned}\right.
$$

Intensive numerical simulations are performed to quantify the effects of various strategies in the Bangalore urban and Chennai districts. The fixed parameters and initial conditions are taken from Tables 1 and 2, respectively. The initial number of protected/vaccinated people is estimated using vaccination coverage data from both the districts [7]. Estimated parameters for both districts are mentioned in the model calibration section. Using these parameters and control parameters at various levels we simulate the model (10) with 3 months ahead of projections.

For both the districts, the projection period is 1 November 2021 to 31 January 2022. To quantify the effects of single control strategies more precisely, we calculate the percentage reduction of notified and hospitalized persons in the 3 month projection period. We use the following basic formula

$$
\begin{aligned}
& \text { Percentage reduction } \\
& =\frac{\text { Baseline cases }- \text { Cases with control }}{\text { Baseline cases }} \times 100 \text {. }
\end{aligned}
$$

The percentage reduction in notified and hospitalized cases are reported in Table 3 for Bangalore urban and Chennai districts. We can see that the face maskrelated controls when applied individually have a moderate impact on the notified and hospitalized populations of both locations. On the other hand, treatment of hospitalized population has a large positive impact on the percentage reduction of hospitalized patients. However, treatment does not show any impact on the notified patients for both the locations. This might be due to the fact that the hospitalized patients are not responsible for new infections directly. Comparing the vaccination rates of susceptible and recovered people, it can be inferred from the table that vaccinating susceptibles has a better impact than that of recovered people. At least in the short-term (3 months) scenario, it is beneficial to vaccinate susceptible people than to vaccinate recovered people. Furthermore, while evaluating the effects of immigration, we noticed that a high level of immigration has a significant negative impact on the percentage reduction. Thus, the immigration of un-notified COVID-19 patients has to be as low as possible. This can be done by allowing peo- 
Table 3 Percentage reduction in the total number of notified and hospitalized COVID-19 patients for different levels of interventions

\begin{tabular}{|c|c|c|c|c|}
\hline \multirow[t]{2}{*}{ Parameter values } & \multicolumn{2}{|l|}{ Bangalore urban } & \multicolumn{2}{|l|}{ Chennai } \\
\hline & Reduction in $I_{n}$ & Reduction in $I_{h}$ & Reduction in $I_{n}$ & Reduction in $I_{h}$ \\
\hline$\epsilon_{m}=0.38$ & 7.47 & 6.98 & 7.49 & 7.19 \\
\hline$=0.55$ & 11.63 & 10.87 & 11.65 & 11.18 \\
\hline$=0.95$ & 20.42 & 19.11 & 20.42 & 19.61 \\
\hline$c_{m}=0.25$ & 4.10 & 3.83 & 4.12 & 3.95 \\
\hline$=0.50$ & 10.43 & 9.75 & 10.45 & 10.03 \\
\hline$=0.75$ & 16.19 & 15.14 & 16.20 & 15.55 \\
\hline$\epsilon_{t}=1.10$ & -0.00 & 8.89 & -0.00 & 8.90 \\
\hline$=1.20$ & -0.00 & 16.30 & -0.00 & 16.31 \\
\hline$=1.30$ & -0.00 & 22.58 & -0.00 & 22.59 \\
\hline$\xi_{s}=0.005$ & 20.86 & 19.31 & 19.87 & 18.98 \\
\hline$=0.01$ & 33.82 & 31.53 & 32.22 & 30.90 \\
\hline$=0.05$ & 60.44 & 56.96 & 58.85 & 56.71 \\
\hline$\xi_{r}=0.01$ & 1.46 & 1.32 & 1.00 & 0.94 \\
\hline$=0.03$ & 2.92 & 2.65 & 2.00 & 1.89 \\
\hline$=0.05$ & 8.85 & 8.10 & 6.22 & 5.90 \\
\hline$\Lambda=1$ & -1.42 & -1.44 & -0.71 & -0.68 \\
\hline$=5$ & -7.10 & -7.19 & -3.57 & -3.39 \\
\hline$=10$ & -14.19 & -14.39 & -7.14 & -6.78 \\
\hline
\end{tabular}

ple from low incidence neighbour districts and restricting immigration from highly affected areas. From the single control results, it is evident that pharmaceutical mitigation strategies like treatment of hospitalized patients and vaccination of susceptible individuals are most effective. Additionally, non-pharmaceutical measures such as face mask use have minimal effects on the reduction of notified and hospitalized COVID-19 cases.

To investigate the combined effects of control interventions, we examine four different combinations on the total number of hospitalized patients $\left(I_{h}^{\text {tot }}\right)$ in the 3 -month projection period. The parameter values are taken as mentioned earlier in this section. We draw contour plots with respect to the control parameters with response variable $I_{h}^{\text {tot }}=\int_{t=289}^{381} I_{h}(t) \mathrm{d} t$. Contour plots depicting combination of interventions are displayed in Figs. 6 and 7 for Bangalore urban and Chennai districts, respectively.

For both of the locations, similar scenarios are seen with varying control interventions and immigration of un-notified infectious patients. Simultaneously increasing face mask efficacy and population-wide compliance level will decrease $I_{h}^{\text {tot }}$ in the 3 month projection period (Figs. 6A, 7A). Thus, both the face mask-related parameters will have a stronger impact on the reduction of $I_{h}^{\text {tot }}$. On the other hand, the vaccination rate of susceptibles and increase in the treatment rate of hospitalized patients will have similar effects for both the locations (as seen in Figs. 6B, 7B). Further, the immigration of un-notified COVID-19 patients will obviously show an increase in $I_{h}^{\text {tot }}$. But, keeping $\Lambda$ low and increasing treatment rate simultaneously will keep $I_{h}^{\text {tot }}$ under control (see Figs. 6C, 7C). From the number of $I_{h}^{t o t}$, it should be noted that this combination is risky if there is no other intervention in action. However, if the vaccination of susceptibles is performed at an increased rate, then the immigration may not have very adverse effects (see Fig. 6D and 7D). It can be noted that a high level of face mask use with maximum efficacy is more effective than the other combined strategies in terms of reduction in $I_{h}^{\text {tot }}$. But the feasibility of face mask usage in such intensity may be critical. From the contour plot analysis, it can be argued that the combination of $\xi_{s}$ and $\epsilon_{t}$ is more feasible and has competitive effects on $I_{h}^{\text {tot }}$. Further numerical simulations are necessary to study the combination of more than two control interventions and the immigration of un-notified infectives.

Three or more control interventions and the immigration of infectives are now applied to examine their combined impact on the COVID-19 cases in the 3 months projection period. We calculate the percentage reduction in notified COVID-19 patients and hospitalized COVID-19 patients using the formula (11). The fixed parameters and initial conditions are taken from Tables 1 and 2 . Four different combination strategies are investigated namely, strategy I: face mask efficacycommunity level usage of face masks-vaccination of susceptibles $\left(\epsilon_{m}-c_{m}-\xi_{s}\right)$, strategy II: face mask efficacycommunity level usage of face masks-vaccination of susceptibles-increase in treatment rate $\left(\epsilon_{m}-c_{m}-\xi_{s}-\epsilon_{t}\right)$, strategy III: face mask efficacy-community level usage of face masks-vaccination of susceptibles-increase in treatment rate-immigration of un-notified infectives $\left(\epsilon_{m}-c_{m}-\xi_{s}-\epsilon_{t}-\Lambda\right)$ and strategy IV: combination of all control interventions with immigration of un-notified infectives $\left(\epsilon_{m}-c_{m}-\xi_{s}-\epsilon_{t}-\Lambda-\xi_{r}\right)$. The parameter values are chosen for the feasibility of the strategies, i.e., we do not consider hypothetically high values of the parame- 
(A)

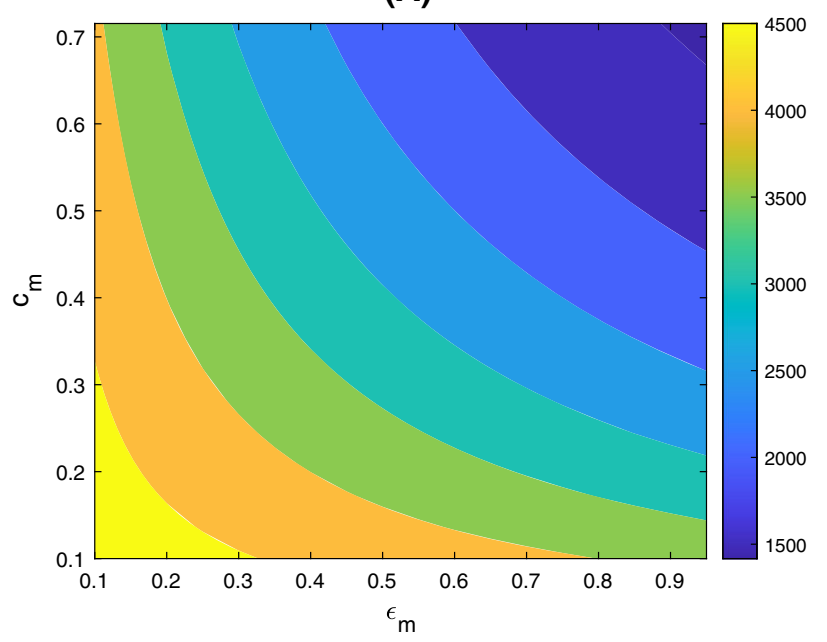

(C)

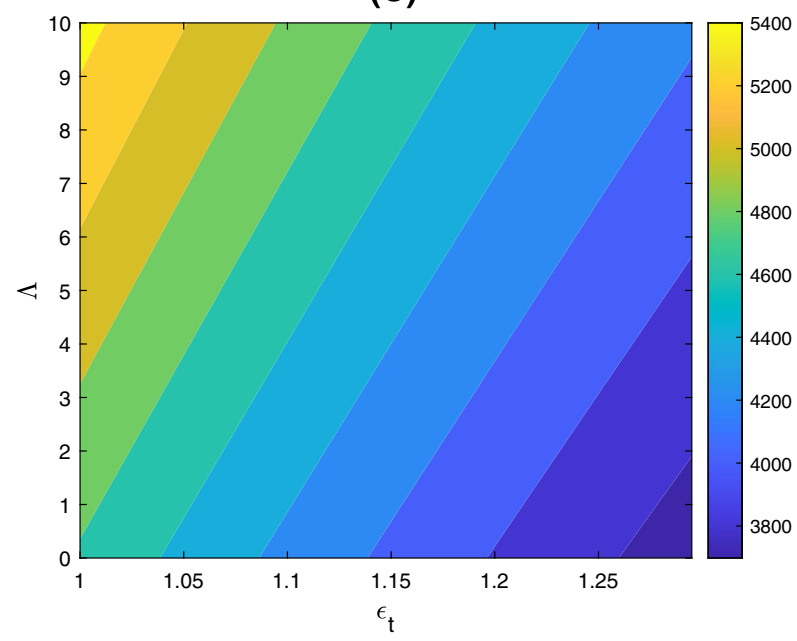

Fig. 6 Combination of control strategies and immigration of infectives in Bangalore urban. A efficacy of face mask usage - community-wide compliance in face mask usage $\left(\epsilon_{m}-c_{m}\right)$, $\mathbf{B}$ increase in recovery rate of hospitalized patients - vaccination rate of susceptible individu-

ters. The percentage reduction of $I_{n}$ and $I_{h}$ for Bangalore urban and Chennai are reported in Table 4.

From the combinations of strategy I and strategy II, we observe that the second case of strategy II has most the positive reduction for both the districts. However, the travel restrictions are being eased and more people tend to come to these districts. Thus, the immigration of un-notified infectives is also evident and hence it is necessary to consider non-zero values of $\Lambda$. It can be observed that strategy IV (first case) with specified control parameter values and low immigration has the most feasible results on both the locations (marked bold in Table 4). Alternatively, the second case of strategy II shows a similar case reduction in both the locations. This strategy does not include immigration and therefore is not feasible. It can be seen that the second case of strategy III also shows competitive results in terms

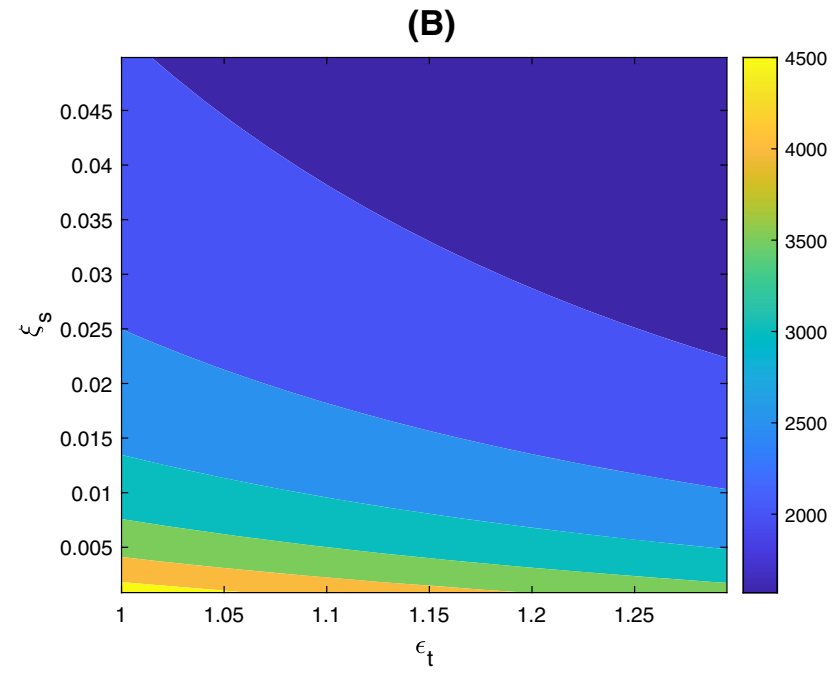

(D)

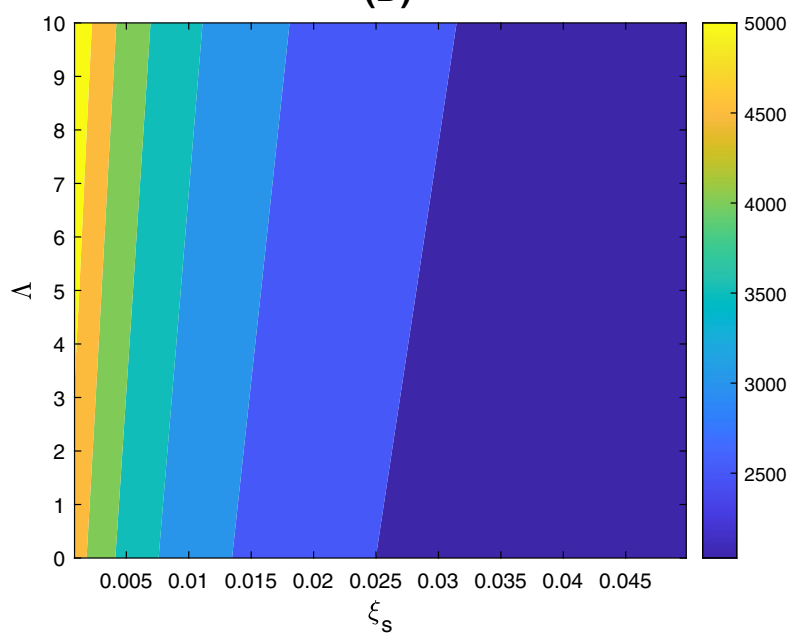

als $\left(\epsilon_{t}-\xi_{s}\right), \mathbf{C}$ increase in recovery rate of hospitalized patients - immigration of infectives $\left(\epsilon_{t}-\Lambda\right)$, D vaccination rate of susceptible individuals - immigration of infectives $\left(\epsilon_{s}-\Lambda\right)$

of percentage reduction of both populations. This strategy is feasible and can be implemented with less effort by the policy makers.

\section{Results and conclusions}

An SEIR-type compartmental model for the transmission dynamics of COVID-19 outbreak incorporating free virus concentration of the environment is considered in this paper. The model assumes standard incidence function while COVID-19 transmission by un-notified and notified individuals. Mass action incidence is considered for the airborne transmission of the virus. The mathematical model consists of a sevendimensional system of ordinary differential equations. 
(A)

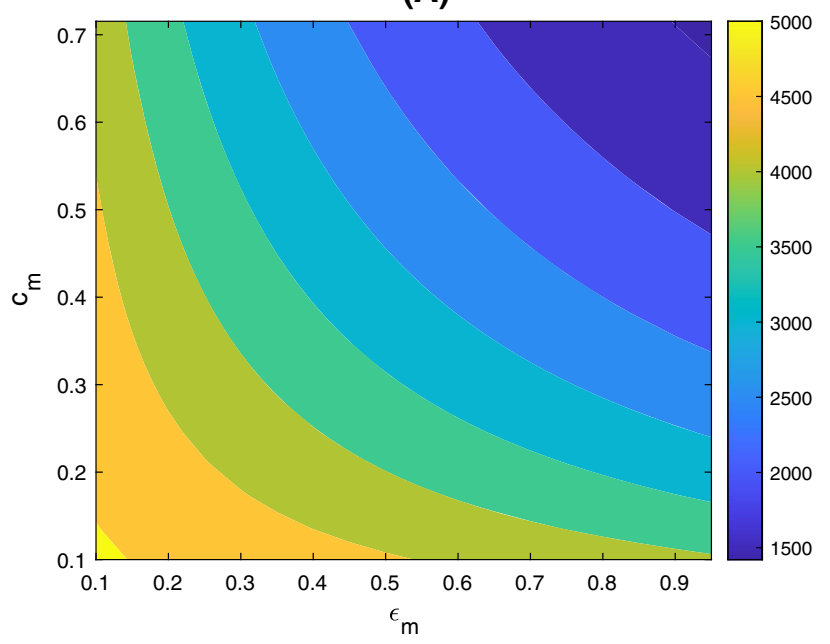

(C)

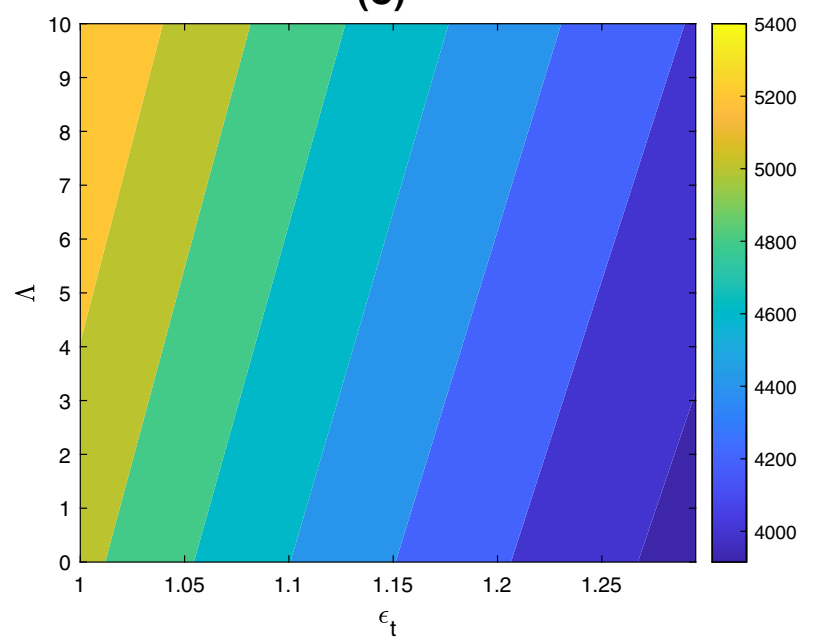

Fig. 7 Combination of control strategies and immigration of infectives in Chennai. A efficacy of face mask usage - community-wide compliance in face mask usage $\left(\epsilon_{m}-c_{m}\right), \mathbf{B}$ increase in recovery rate of hospitalized

The model is mathematically analysed to obtain insight into the long-term dynamic features of the disease. Basic properties of the model are studied by finding positivity and boundedness of the solutions when the initial conditions are taken non-negative for all the state variables. The disease-free equilibrium (DFE) of the model is found to be unique and the basic reproduction number is found using the next-generation matrix method. The model has a locally stable DFE whenever the basic reproduction number is less than unity otherwise the DFE becomes unstable. Moreover, the global stability of the DFE is guaranteed when $\mathscr{R}_{0}<1$. This can be inferred that COVID-19 will be mitigated from the community if the corresponding parameters are in the range to ensure $\mathscr{R}_{0}<1$. The existence of the endemic equilibrium is investigated and it is found that at most two endemic equilibria may exist for the system. Forward transcritical bifurcation is established
(B)

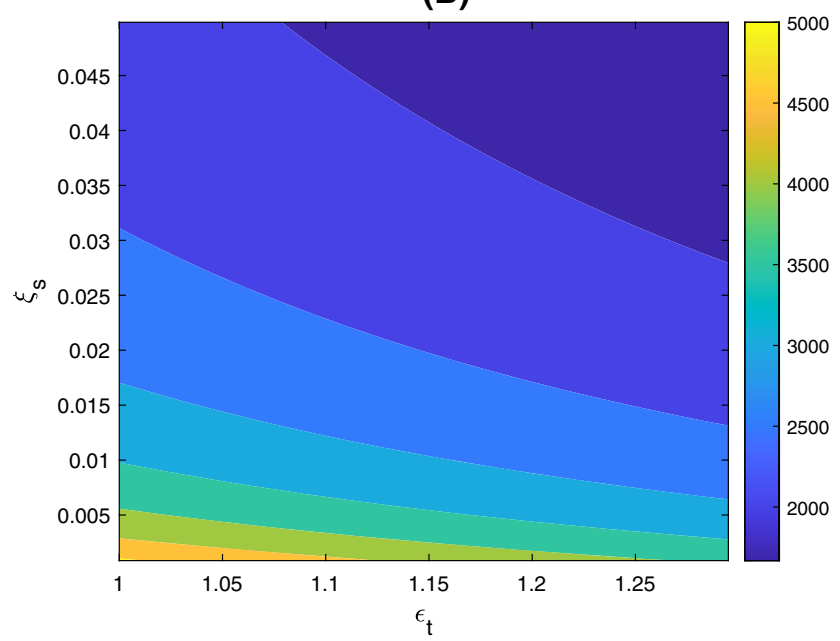

(D)

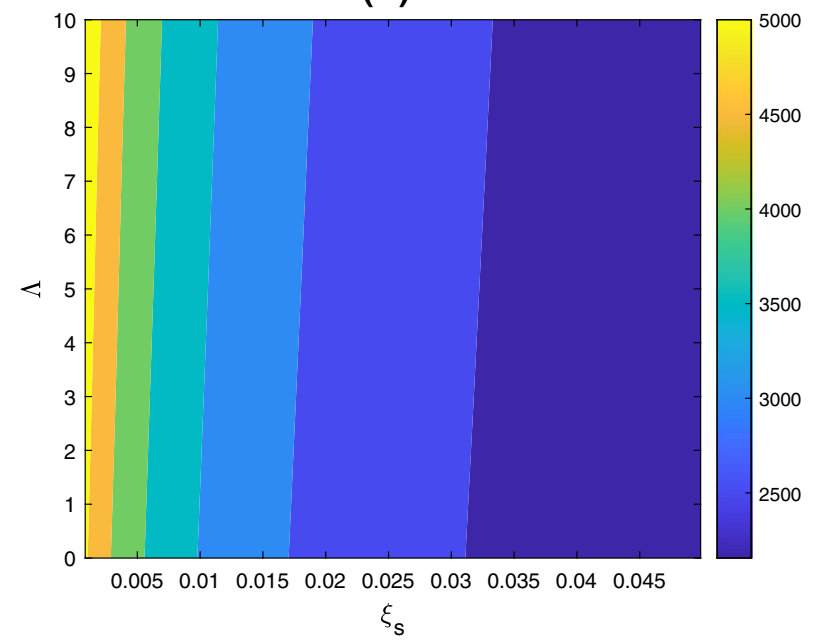

patients - vaccination rate of susceptible individuals $\left(\epsilon_{t}-\right.$ $\left.\xi_{s}\right), \mathbf{C}$ increase in recovery rate of hospitalized patientsimmigration of infectives $\left(\epsilon_{t}-\Lambda\right), \mathbf{D}$ vaccination rate of susceptible individuals - immigration of infectives $\left(\epsilon_{s}-\Lambda\right)$

both analytically and numerically between DFE and one of the endemic equilibrium (see Fig. 2). Further, it is numerically shown that all the infected compartments tend to vanish in the long run when $\mathscr{R}_{0}<1$ and approaches a non-zero equilibrium when $\mathscr{R}_{0}>1$ (shown in Fig. 3).

We calibrated the proposed model parameters to fit daily mortality data of two districts of India during the period of January $16^{t h}, 2021$ to October $31^{s t}, 2021$. From the fitting of the model output to mortality data, it can be seen that the model output closely anticipates the real data for both the districts. Afterwards we investigate the impacts of control strategies and immigration of un-notified persons on the number of hospitalized patients. We concentrate on both pharmaceutical and non-pharmaceutical control measures as they are being implemented simultaneously to combat the deadly disease. Two pharmaceutical measures 
Table 4 Percentage reduction in total number of notified and hospitalized COVID-19 patients for different strategies

\begin{tabular}{|c|c|c|c|c|c|}
\hline & \multirow[t]{2}{*}{ Parameter values } & \multicolumn{2}{|l|}{ Bangalore urban } & \multicolumn{2}{|l|}{ Chennai } \\
\hline & & Reduction in $I_{n}$ & Reduction in $I_{h}$ & Reduction in $I_{n}$ & Reduction in $I_{h}$ \\
\hline \multirow[t]{3}{*}{ Strategy-I } & $\begin{array}{l}\epsilon_{m}=0.38, c_{m}=0.25, \xi_{s}= \\
0.005\end{array}$ & 33.67 & 31.47 & 32.85 & 31.54 \\
\hline & $\begin{array}{l}\epsilon_{m}=0.38, c_{m}=0.50, \xi_{s}= \\
0.005\end{array}$ & 44.77 & 42.02 & 44.09 & 42.42 \\
\hline & $\begin{array}{l}\epsilon_{m}=0.55, c_{m}=0.25, \xi_{s}= \\
0.005\end{array}$ & 39.00 & 36.53 & 38.25 & 36.76 \\
\hline \multirow[t]{3}{*}{ Strategy-II } & $\begin{array}{l}\epsilon_{m}=0.38, c_{m}=0.25, \xi_{s}= \\
0.005, \epsilon_{t}=1.1\end{array}$ & 33.67 & 37.54 & 32.85 & 37.60 \\
\hline & $\begin{array}{l}\epsilon_{m}=0.38, c_{m}=0.50, \xi_{s}= \\
0.005, \epsilon_{t}=1.1\end{array}$ & 44.77 & 47.14 & 44.09 & 47.49 \\
\hline & $\begin{array}{l}\epsilon_{m}=0.55, c_{m}=0.25, \xi_{s}= \\
0.005, \epsilon_{t}=1.1\end{array}$ & 39.00 & 42.15 & 38.25 & 42.35 \\
\hline \multirow[t]{6}{*}{ Strategy-III } & $\begin{array}{l}\epsilon_{m}=0.38, c_{m}=0.25, \xi_{s}= \\
0.005, \epsilon_{t}=1.1, \Lambda=5\end{array}$ & 29.78 & 33.63 & 30.91 & 35.91 \\
\hline & $\begin{array}{l}\epsilon_{m}=0.38, c_{m}=0.50, \xi_{s}= \\
0.005, \epsilon_{t}=1.1, \Lambda=5\end{array}$ & 41.62 & 43.86 & 42.52 & 46.12 \\
\hline & $\begin{array}{l}\epsilon_{m}=0.55, c_{m}=0.25, \xi_{s}= \\
0.005, \epsilon_{t}=1.1, \Lambda=5\end{array}$ & 35.46 & 38.53 & 36.48 & 40.81 \\
\hline & $\begin{array}{l}\epsilon_{m}=0.38, c_{m}=0.25, \xi_{s}= \\
0.005, \epsilon_{t}=1.1, \Lambda=10\end{array}$ & 25.89 & 29.72 & 28.97 & 34.22 \\
\hline & $\begin{array}{l}\epsilon_{m}=0.38, c_{m}=0.50, \xi_{s}= \\
0.005, \epsilon_{t}=1.1, \Lambda=10\end{array}$ & 38.47 & 40.57 & 40.94 & 44.75 \\
\hline & $\begin{array}{l}\epsilon_{m}=0.55, c_{m}=0.25, \xi_{s}= \\
0.005, \epsilon_{t}=1.1, \Lambda=10\end{array}$ & 31.91 & 34.91 & 34.71 & 39.27 \\
\hline \multirow[t]{2}{*}{ Strategy-IV } & $\begin{array}{l}\epsilon_{m}=0.38, c_{m}=0.50, \xi_{s}= \\
0.005, \xi_{r}=0.01, \epsilon_{t}=1.1, \Lambda \\
=5\end{array}$ & 42.31 & 44.44 & 42.92 & 46.48 \\
\hline & $\begin{array}{l}\epsilon_{m}=0.38, c_{m}=0.50, \xi_{s}= \\
0.005, \xi_{r}=0.01, \epsilon_{t}=1.1, \Lambda \\
=10\end{array}$ & 39.30 & 41.27 & 41.40 & 45.15 \\
\hline
\end{tabular}

The most feasible strategies for both the locations are marked in bold

namely, treatment of hospitalized persons and vaccination of susceptibles as well as recovered persons are considered. On the other hand, face mask efficacy and community-wide compliance of face masks are considered as non-pharmaceutical control measures. Along with these interventions, we also study the effect of immigrating un-notified persons from other communities. We calculated the percentage reduction in total hospitalized patients to get a quantitative idea of the control measures and immigration. It is found that treatment of hospitalized patients and vaccination of susceptible impacts has a significant impact on the reduction of hospitalized cases (see Table 3 ). Therefore, pharmaceutical measures are more reliable than nonpharmaceutical controls. Additionally, immigration of un-notified infected persons may drive the hospitalized cases to higher prevalence. It is noted that face maskrelated controls have a lower impact than pharmaceutical measures like treatment and vaccination. The immigration of un-notified patients has a negative impact on the total hospitalized cases and thus this should be kept as low as possible. Low immigration of infectives can be maintained by restricting immigration from highly affected neighbouring districts. Further, we investigate the combined effect of two strategies on the total number of hospitalized patients for both districts. We found that the combination of treatment and vaccination of susceptible individuals will be very effective to reduce the disease. Finally, we compare three or more interventions by calculating the percentage reduction of notified and hospitalized patients with different levels of feasible control measures and immigration rates. We examine strategy I-strategy IV (as defined in the previous section) and find that the combination of all controls with low immigration will have the best effect on the reduction of the disease. However, the second case of strategy III also shows competitive results in terms of percentage reduction of both populations. This strategy differs from strategy IV by the vaccination of recovered people. Therefore, it can be concluded that when vaccine crisis is there, the public health authorities may not vaccinate the recovered people. In other words, the peo- 
ple who were tested COVID-19 positive in past and are now healthy (they may have developed natural antibodies against SARS-CoV-2) may be vaccinated later when vaccines are available. Low immigration of un-notified patients can be regulated by restricting the movement of people from high prevalence adjacent districts. This analysis can be made with data from other profoundly affected districts with proper parametrization.

In summary, the results indicate that vaccination of susceptible individuals and treatment of hospitalized patients are very crucial to control COVID-19 in the two locations. In the 3 months short-term period, vaccination of susceptible people should be prioritized over vaccination of recovered people for a better outcome. Whenever vaccine crisis is there, governments may avoid the vaccination of people who were tested COVID-19 positive and are now healthy. However, increased quality and quantity of mask use are also helpful. Along with face mask use, treatment of hospitalized patients, and vaccination of susceptibles, immigration should be allowed in a supervised manner so that economy of the overall society remains healthy.

Acknowledgements The authors are grateful to the learned reviewer for commenting on the earlier version of this manuscript which have immensely improve the standard of the paper. Research of Indrajit Ghosh is supported by National Board for Higher Mathematics (NBHM) postdoctoral fellowship (Ref. No: 0204/3/2020/R \& D-II/2458), Department of Atomic Energy, Government of India.

Data Availability Statement This manuscript has associated data in a data repository. [Authors' comment: The data are taken from the website https://www.covid19india. $\operatorname{org} /$.

\section{Declarations}

Conflict of interest The authors declare that they have no conflict of interest.

\section{References}

1. S. Addleman, V. Leung, L. Asadi, A. Sharkawy, J. McDonald, Mitigating airborne transmission of SARSCoV-2. CMAJ 193(26), E1010-E1011 (2021)

2. M.A. Andrews, B. Areekal, K.R. Rajesh, J. Krishnan, R. Suryakala, B. Krishnan, C.P. Muraly, P.V. Santhosh, First confirmed case of COVID-19 infection in India: a case report. Indian J. Med. Res. 151(5), 490 (2020)

3. J. Bere, N.D. Jonnalagadda, L. Kappari, J. Karangula, N. Boggula, V. Kappari, 2-Deoxy-D-glucose: an update review. J. Innov. Dev. Pharm. Tech. Sci. (JIDPTS) 4(5), 68-78 (2021)

4. C. Castillo-Chavez, Z. Feng, W. Huang et al., On the computation of $\mathscr{R}_{0}$ and its role in global stability, in mathematical approaches for emerging and reemerging infectious diseases: an introduction (Minneapolis, MN,
1999). IMA Volumes in Mathematics and Its Applications, vol 125 (Springer, New York, 2002), p. 229-250

5. C. Castillo-Chavez, B. Song, Dynamical models of tuberculosis and their applications. Math. Biosci. Eng. 1(2), 361-404 (2004)

6. Coronavirus worldometer (2021), https://www. worldometers.info/coronavirus/. Accessed 22 Nov 2021

7. covid19india (2021), https://www.covid19india.org/. Accessed 22 Nov 2021

8. Demographics worldometer (2021), https://www. worldometers.info/demographics/india-demographics/. Accessed 22 Nov 2021

9. O. Diekmann, J.A.P. Heesterbeek, J.A.J. Metz, On the definition and the computation of the basic reproduction ratio $\mathscr{R}_{0}$ in models for infectious diseases in heterogeneous populations. J. Math. Biol. 28(4), 365-382 (1990)

10. D. Easwaramoorthy, A. Gowrisankar, A. Manimaran, S. Nandhini, L. Rondoni, S. Banerjee, An exploration of fractal-based prognostic model and comparative analysis for second wave of COVID-19 diffusion. Nonlinear Dyn. 106(2), 1375-1395 (2021)

11. Editorial, COVID-19 transmission-up in the air. Lancet Respir. Med. 8(12), 1159 (2020)

12. B.H. Foy, B. Wahl, K. Mehta, A. Shet, G.I. Menon, C. Britto, Comparing COVID-19 vaccine allocation strategies in India: a mathematical modelling study. Int. J. Infect. Dis. 103, 431-438 (2021)

13. I. Ghosh, M. Martcheva, Modeling the effects of prosocial awareness on COVID-19 dynamics: case studies on Colombia and India. Nonlinear Dyn. 104, 4681-4700 (2021)

14. S. Ghosh, A. Senapati, J. Chattopadhyay, C. Hens, D. Ghosh, Optimal test-kit-based intervention strategy of epidemic spreading in heterogeneous complex networks. Chaos Interdiscip. J. Nonlinear Sci. 31(7), 071101 (2021)

15. A. Gowrisankar, L. Rondoni, S. Banerjee, Can India develop herd immunity against COVID-19? Eur. Phys. J. Plus 135(6), 1-9 (2020)

16. T. Greenhalgh, J.L. Jimenez, K.A. Prather, Z. Tufekci, D. Fisman, R. Schooley, Ten scientific reasons in support of airborne transmission of SARS-CoV-2. Lancet 397(10285), 1603-1605 (2021)

17. C. Kavitha, A. Gowrisankar, S. Banerjee, The second and third waves in India: when will the pandemic be culminated? Eur. Phys. J. Plus 136(5), 596 (2021)

18. W.O. Kermack, A.G. McKendrick, A contribution to the mathematical theory of epidemics. Proc. R. Soc. Math. Phys. Eng. Sci. 115(772), 700-721 (1927)

19. S. Khajanchi, K. Sarkar, Forecasting the daily and cumulative number of cases for the COVID-19 pandemic in India. Chaos Interdiscip. J. Nonlinear Sci. 30(7), $071101(2020)$

20. H. Lau, T. Khosrawipour, P. Kocbach, H. Ichii, J. Bania, V. Khosrawipour, Evaluating the massive underreporting and undertesting of COVID-19 cases in multiple global epicenters. Pulmonology 27(2), 110-115 (2021)

21. Q. Li, X. Guan, P. Wu, X. Wang, L. Zhou, Y. Tong, R. Ren, K.S.M. Leung, E.H.Y. Lau, J.Y. Wong et al., Early transmission dynamics in Wuhan, China, of novel coronavirus-infected pneumonia. N. Engl. J. Med. 382, 1199-1207 (2020) 
22. N.M. Linton, T. Kobayashi, Y. Yang, K. Hayashi, A.R. Akhmetzhanov, S. Jung, B. Yuan, R. Kinoshita, H. Nishiura, Incubation period and other epidemiological characteristics of 2019 novel coronavirus infections with right truncation: a statistical analysis of publicly available case data. J. Clin. Med. 9(2), 538 (2020)

23. Y. Liu, L.-M. Yan, L. Wan, T.-X. Xiang, A. Le, J.-M. Liu, M. Peiris, L.L.M. Poon, W. Zhang, Viral dynamics in mild and severe cases of COVID-19. Lancet Infect. Dis. 20(6), 656-657 (2020)

24. L. López, X. Rodó, The end of social confinement and COVID-19 re-emergence risk. Nat. Hum. Behav. 4(7), 746-755 (2020)

25. Macrotrends Bangalore Population (2021), https:// www.macrotrends.net/cities/21176/bangalore/ population. Accessed 22 Nov 2021

26. Macrotrends Chennai Population (2021), https://www. macrotrends.net/cities/21321/madras/population. Accessed 22 Nov 2021

27. M. Mandal, S. Jana, S.K. Nandi, A. Khatua, S. Adak, T.K. Kar, A model based study on the dynamics of COVID-19: prediction and control. Chaos Solitons Fractals 136, 109889 (2020)

28. S. Mandal, T. Bhatnagar, N. Arinaminpathy, A. Agarwal, A. Chowdhury, M. Murhekar, R.R. Gangakhedkar, S. Sarkar, Prudent public health intervention strategies to control the coronavirus disease 2019 transmission in India: a mathematical model-based approach. Indian J. Med. Res. 151(2-3), 190 (2020)

29. M. Martcheva, An Introduction to Mathematical Epidemiology, vol. 61 (Springer, New York, 2015)

30. C.N. Ngonghala, E.A. Iboi, A.B. Gumel, Could masks curtail the post-lockdown resurgence of COVID-19 in the US? Math. Biosci. 329, 108452 (2020)

31. PIB. DCGI approves anti-COVID drug developed by DRDO for emergency use. https://pib.gov.in/ PressReleasePage.aspx?PRID $=1717007,2021$. Press Information Bureau, Government of India. 2021-05-08 Retrieved: 2021-11-22

32. F. Saldaña, H. Flores-Arguedas, J.A. CamachoGutiérrez, I. Barradas, Modeling the transmission dynamics and the impact of the control interventions for the COVID-19 epidemic outbreak. Math. Biosci. Eng. 17(4), 4165-4183 (2020)

33. K.C. Samal, B. Panda, L. Behera, Anti-COVID drug: 2deoxy-D-glucose and its mechanism of action. Biot. Res. Today 3(5), 345-347 (2021)

34. T. Sardar, S.S. Nadim, S. Rana, J. Chattopadhyay, Assessment of lockdown effect in some states and overall India: a predictive mathematical study on COVID-19 outbreak. Chaos Solitons Fractals 139, 110078 (2020)

35. A. Sariol, S. Perlman, Lessons for COVID-19 immunity from other coronavirus infections. Immunity 53(2), 248263 (2020)

36. A. Senapati, S. Rana, T. Das, J. Chattopadhyay, Impact of intervention on the spread of COVID-19 in India: a model based study. J. Theor. Biol. 523, 110711 (2021)

37. S.K. Sharma, M. Mishra, S.K. Mudgal, Efficacy of cloth face mask in prevention of novel coronavirus infection transmission: a systematic review and meta-analysis. J. Educ. Health Promot. 9, 192 (2020)

38. K. Sharun, K. Dhama, India's role in COVID-19 vaccine diplomacy. J. Travel Med. 28(7), 1-4 (2021)
39. A.S. Soin, K. Kumar, N.S. Choudhary, P. Sharma, Y. Mehta, S. Kataria, D. Govil, V. Deswal, D. Chaudhry, P.K. Singh et al., Tocilizumab plus standard care versus standard care in patients in India with moderate to severe COVID-19-associated cytokine release syndrome (COVINTOC): an open-label, multicentre, randomised, controlled, phase 3 trial. Lancet Respir. Med. 9(5), 511$521(2021)$

40. R.O.J.H. Stutt, R. Retkute, M. Bradley, C.A. Gilligan, J. Colvin, A modelling framework to assess the likely effectiveness of facemasks in combination with 'lockdown' in managing the COVID-19 pandemic. Proc. R. Soc. Math. Phys. Eng. Sci. 476(2238), 20200376 (2020)

41. F.V. Surano, M. Porfiri, A. Rizzo, Analysis of lockdown perception in the United States during the COVID-19 pandemic. Eur. Phys. J. Spec. Top. 1-9 (2021). https:// doi.org/10.1140/epjs/s11734-021-00265-z

42. L. Tindale, M. Coombe, J.E. Stockdale, E. Garlock, W.Y.V. Lau, M. Saraswat, Y.-H.B. Lee, L. Zhang, D. Chen, J. Wallinga et al., Transmission interval estimates suggest pre-symptomatic spread of COVID19. MedRxiv (2020). https://doi.org/10.1101/2020.03. 03.20029983

43. P. van den Driessche, J. Watmough, Reproduction numbers and sub-threshold endemic equilibria for compartmental models of disease transmission. Math. Biosci. 180(1-2), 29-48 (2002)

44. M. van der Sande, P. Teunis, R. Sabel, Professional and home-made face masks reduce exposure to respiratory infections among the general population. PLoS One 3(7), e2618 (2008)

45. N. van Doremalen, T. Bushmaker, D.H. Morris, M.G. Holbrook, A. Gamble, B.N. Williamson, A. Tamin, J.L. Harcourt, N.J. Thornburg, S.I. Gerber et al., Aerosol and surface stability of SARS-CoV-2 as compared with SARS-CoV-1. N. Engl. J. Med. 382(16), 1564-1567 (2020)

46. WHO, Coronavirus disease 2019 (COVID-19): situation report, 73 (2020), https://apps.who.int/iris/handle/ $10665 / 331686$

47. WHO, Rolling updates on coronavirus disease (COVID19): who characterizes COVID-19 as a pandemic (2020), https://www.who.int/emergencies/diseases/ novel-coronavirus-2019/events-as-they-happen

48. R. Woelfel, V.M. Corman, W. Guggemos, M. Seilmaier, S. Zange, M.A. Mueller, D. Niemeyer, P. Vollmar, C. Rothe, M. Hoelscher, et al., Clinical presentation and virological assessment of hospitalized cases of coronavirus disease 2019 in a travel-associated transmission cluster. MedRxiv (2020). https://doi.org/10.1101/2020. 03.05.20030502

49. J.T. Wu, K. Leung, G.M. Leung, Nowcasting and forecasting the potential domestic and international spread of the 2019-nCoV outbreak originating in Wuhan, China: a modelling study. Lancet 395(10225), 689-697 (2020)

50. Z. Wu, J.M. McGoogan, Characteristics of and important lessons from the coronavirus disease 2019 (COVID19) outbreak in China: summary of a report of 72314 cases from the Chinese Center for Disease Control and Prevention. JAMA 323(13), 1239-1242 (2020) 
51. X. Xiao-Wei, W. Xiao-Xin, X.-G. Jiang, X. Kai-Jin, L.J. Ying, C.-L. Ma, S.-B. Li, H.-Y. Wang, S. Zhang, H.N. Gao et al., Clinical findings in a group of patients infected with the 2019 novel coronavirus (SARS-CoV2) outside of Wuhan, China: retrospective case series. BMJ 368, m606 (2020)
52. P. Yang, Y. Ding, X. Zhe, P. Rui, P. Li, J. Yan, J. Liu, F. Meng, L. Huang, L. Shi et al., Epidemiological and clinical features of COVID-19 patients with and without pneumonia in Beijing (China). MedRxiv (2020). https://doi.org/10.1101/2020.02.28.20028068 